\title{
Uniform attractors for the non-autonomous $p$-Laplacian equations with dynamic flux boundary conditions
}

Kun $\mathrm{Li}^{1}$ and Bo You ${ }^{2 *}$

${ }^{*}$ Correspondence: youb03@126.com

${ }^{2}$ School of Mathematics and

Statistics, Xi'an Jiaotong University, Xi'an, 710049, P.R. China

Full list of author information is

available at the end of the article

\begin{abstract}
This paper studies the long-time asymptotic behavior of solutions for the non-autonomous $p$-Laplacian equations with dynamic flux boundary conditions in $n$-dimensional bounded smooth domains. We have proved the existence of the uniform attractor in $L^{2}(\bar{\Omega}, d \mu)$ for the non-autonomous $p$-Laplacian evolution equations subject to dynamic nonlinear boundary conditions by using the Sobolev compactness embedding theory, and the existence of the uniform attractor in $\left(W^{1, p}(\Omega) \cap L^{q}(\Omega)\right) \times L^{q}(\Gamma)$ by asymptotic a priori estimate.
\end{abstract}

\section{Introduction}

We are concerned with the existence of uniform attractors for the process associated with the solutions of the following non-autonomous $p$-Laplacian equation:

$$
u_{t}-\Delta_{p} u+|u|^{p-2} u+f(u)=g(x, t), \quad(x, t) \in \Omega \times \mathbb{R} .
$$

Equation (1) is subject to the dynamic flux boundary condition

$$
u_{t}+|\nabla u|^{p-2} \frac{\partial u}{\partial v}+f(u)=0, \quad(x, t) \in \Gamma \times \mathbb{R}
$$

and the initial condition

$$
u(x, \tau)=u_{0}(x), \quad x \in \bar{\Omega},
$$

where $\Omega \subset \mathbb{R}^{n}(n \geq 3)$ is a bounded domain with smooth boundary $\Gamma, v$ denotes the outer unit normal on $\Gamma, p \geq 2$, the nonlinearity $f$ and the external force $g$ satisfy some conditions specified later.

Non-autonomous equations appear in many applications in the natural sciences, so they are of great importance and interest. The long-time behavior of solutions of such equations has been studied extensively in recent years (e.g., see [1-4]). The first attempt was to extend the notion of a global attractor to the non-autonomous case, leading to the concept of the so-called uniform attractor (see [5]). It is remarkable that the conditions ensuring the existence of a uniform attractor are parallel with those for the autonomous case. A uniform attractor need not be 'invariant', unlike a global attractor for autonomous

(c) 2013 Li and You; licensee Springer. This is an Open Access article distributed under the terms of the Creative Commons Attribution License (http://creativecommons.org/licenses/by/2.0), which permits unrestricted use, distribution, and reproduction in any medium, provided the original work is properly cited. 
systems. Moreover, it is well known that the trajectories may be unbounded for many nonautonomous systems when the time tends to infinity, and there does not exist a uniform attractor for these systems.

Dynamic boundary conditions are very natural in many mathematical models such as heat transfer in a solid in contact with a moving fluid, thermoelasticity, diffusion phenomena, heat transfer in two mediums, problems in fluid dynamics (see [1-4, 6-11]).

In recent years, many authors have studied $p$-Laplacian equations (see [12-17]) and the problem (1)-(3) for $p=2$ (see $[3,7,9,10]$ ) by discussing the existence and uniqueness of local solutions, the blow-up of solutions, the global existence of solutions, the global attractors of solutions and the eigenvalue problems, etc. In [18], the authors have proved the global existence of solutions for quasi-linear elliptic equations with dynamic boundary conditions. Due to the complications inherent to nonlinear dynamic boundary conditions, these problems (1)-(3) still need to be investigated. In [15-17, 19], the authors have considered the eigenvalue problem

$$
\left\{\begin{array}{l}
-\Delta_{p} u+|u|^{p-2} u=0, \quad x \in \Omega, \\
|\nabla u|^{p-2} \frac{\partial u}{\partial v}=\lambda|u|^{p-2} u, \quad x \in \Gamma
\end{array}\right.
$$

and obtained some results, and some $p$-Laplacian elliptic equations with nonlinear boundary condition have been studied by using these results mentioned in [15-17, 19]. In [14, $20]$, the authors have proved the existence of uniform attractors for the non-autonomous $p$-Laplacian equations with Dirichlet boundary conditions in a bounded and an unbounded domain in $\mathbb{R}^{n}$. The authors have proved the existence of global attractors for the autonomous $p$-Laplacian equations with dynamic flux boundary conditions in [21]. In [11], the authors have used a new type of uniformly Gronwall inequality and proved the existence of a pullback attractor in $L^{r_{1}}(\Omega) \times L^{r_{2}}(\Gamma)$ of the following equation:

$$
\left\{\begin{array}{l}
u_{t}-\Delta_{p} u+|u|^{p-2} u+f(u)=h(t), \quad(x, t) \in \Omega \times \mathbb{R}, \\
u_{t}+|\nabla u|^{p-2} \frac{\partial u}{\partial v}+g(u)=0, \quad(x, t) \in \Gamma \times \mathbb{R}, \\
u(x, \tau)=u_{0}(x), \quad x \in \bar{\Omega},
\end{array}\right.
$$

under the assumptions that $f, g$ satisfy the polynomial growth condition with order $r_{1}, r_{2}$ and $h(t)$ satisfies some weak assumption

$$
\int_{-\infty}^{t} e^{\theta s}\|h(s)\|_{L^{2}(\Omega)}^{2} d s<\infty
$$

for all $t \in \mathbb{R}$, where $\theta$ is some positive constant.

Moreover, the existence of uniform attractors for the non-autonomous $p$-Laplacian equations with dynamical boundary conditions remains unsolvable.

To study problem (1)-(3), we assume the following conditions.

$\left(\mathrm{H}_{1}\right)$ The functions $f \in C^{1}(\mathbb{R}, \mathbb{R})$ and satisfy

$$
f^{\prime}(u) \geq-l
$$


for some $l \geq 0$, and

$$
c_{1}|u|^{q}-k \leq f(u) u \leq c_{2}|u|^{q}+k,
$$

where $c_{i}>0(i=1,2), q>2, k>0$.

$\left(\mathrm{H}_{2}\right)$ The external force $g: \Omega \times \mathbb{R} \rightarrow \mathbb{R}$ is locally Lipschitz continuous, $\frac{d g}{d t}, g \in$ $L_{\text {loc }}^{2}\left(\mathbb{R}, L^{2}(\Omega)\right)$ and satisfies

$$
\sup _{r \in \mathbb{R}} \int_{r}^{r+1}\|g(s)\|_{L^{2}(\Omega)}^{2} d s<\infty
$$

$\left(\mathrm{H}_{3}\right)$ Furthermore, $g(t)$ is uniformly bounded in $L^{2}(\Omega)$ with respect to $t \in \mathbb{R}$, i.e., there exists a positive constant $K$ such that

$$
\sup _{t \in \mathbb{R}}\|g(t)\|_{L^{2}(\Omega)} \leq K
$$

The main purpose of this paper is to study the long-time dynamical behavior for the non-autonomous $p$-Laplacian evolutionary equations (1)-(3) under quite general assumptions (4)-(6). We first prove the existence and the uniqueness of solutions for (1)-(5), and then the existence of uniformly (w.r.t. $\sigma \in \mathcal{H}_{w}(g)$ ) absorbing sets for the process $\left\{U_{\sigma}(t, \tau)\right\}_{\sigma \in \mathcal{H}_{w}(g)}$ corresponding to (1)-(5) in $L^{2}(\bar{\Omega}, d \mu)$ and $\left(W^{1, p}(\Omega) \cap L^{q}(\Omega)\right) \times L^{q}(\Gamma)$, respectively, is obtained. Finally, the existence of the uniform (w.r.t. $\sigma \in \mathcal{H}_{w}(g)$ ) attractor for the process $\left\{U_{\sigma}(t, \tau)\right\}_{\sigma \in \mathcal{H}_{w}(g)}$ corresponding to (1)-(5) in $L^{2}(\bar{\Omega})$ is obtained by the Sobolev compactness embedding theory and the existence of the uniform (w.r.t. $\sigma \in \mathcal{H}_{w}(g)$ ) attractor for the process $\left\{U_{\sigma}(t, \tau)\right\}_{\sigma \in \mathcal{H}_{w}(g)}$ corresponding to (1)-(5) in $\left(W^{1, p}(\Omega) \cap L^{q}(\Omega)\right) \times L^{q}(\Gamma)$ is obtained by asymptotic a priori estimate.

This paper is organized as follows. In Section 2, we give some notations and lemmas used in the sequel. The existence and the uniqueness of solutions for the problem (1)-(5) have been proved in Section 3. Section 4 is devoted to proving the existence of the uniformly (w.r.t. $\sigma \in \mathcal{H}_{w}(g)$ ) absorbing sets in $L^{2}(\bar{\Omega}, d \mu), L^{q}(\bar{\Omega}, d \mu)$ and $\left(L^{q}(\Omega) \cap W^{1, p}(\Omega)\right) \times L^{q}(\Gamma)$, respectively, for the process $\left\{U_{\sigma}(t, \tau)\right\}_{\sigma \in \mathcal{H}_{w}(g)}$ corresponding to (1)-(5) and the existence of the uniform (w.r.t. $\left.\sigma \in \mathcal{H}_{w}(g)\right)$ attractors in $L^{2}(\bar{\Omega}, d \mu), L^{q}(\bar{\Omega}, d \mu)$ and $\left(L^{q}(\Omega) \cap W^{1, p}(\Omega)\right) \times$ $L^{q}(\Gamma)$, respectively, for the process $\left\{U_{\sigma}(t, \tau)\right\}_{\sigma \in \mathcal{H}_{w}(g)}$ corresponding to (1)-(5).

Throughout this paper, we denote the inner product in $L^{2}(\Omega)$ (or $\left.L^{2}(\Gamma)\right)$ by $(\cdot, \cdot)$, and let $C$ be a positive constant, which may be different from line to line (and even in the same line); we denote the trace operator by $\gamma$.

\section{Preliminaries}

In order to study the problem (1)-(5), we recall the Sobolev space $W^{1, p}(\Omega)$ defined as the closure of $C^{\infty}(\Omega) \cap W^{1, p}(\Omega)$ in the norm

$$
\|u\|_{1, p}=\left(\int_{\Omega}|\nabla u|^{p}+|u|^{p} d x\right)^{\frac{1}{p}}
$$

and denote by $X^{*}$ the dual space of $X$. We also define the Lebesgue spaces as follows:

$$
L^{r}(\Gamma)=\left\{v:\|v\|_{L^{r}(\Gamma)}<\infty\right\},
$$


where

$$
\|v\|_{L^{r}(\Gamma)}=\left(\int_{\Gamma}|v|^{r} d S\right)^{\frac{1}{r}}
$$

for $r \in[1, \infty)$. Moreover, we have

$$
L^{s}(\Omega) \oplus L^{s}(\Gamma)=L^{s}(\bar{\Omega}, d \mu), \quad s \in[1, \infty)
$$

and

$$
\|U\|_{L^{s}(\bar{\Omega}, d \mu)}=\left(\int_{\Omega}|u|^{s} d x\right)^{\frac{1}{s}}+\left(\int_{\Gamma}|v|^{s} d S\right)^{\frac{1}{s}}
$$

for any $U=\left(\begin{array}{l}u \\ v\end{array}\right) \in L^{s}(\bar{\Omega}, d \mu)$, where the measure $d \mu=\left.\left.d x\right|_{\Omega} \oplus d S\right|_{\Gamma}$ on $\bar{\Omega}$ is defined for any measurable set $A \subset \bar{\Omega}$ by $\mu(A)=|A \cap \Omega|+S(A \cap \Gamma)$. In general, any vector $\theta \in L^{s}(\bar{\Omega}, d \mu)$ will be of the form $\left(\begin{array}{c}\theta_{1} \\ \theta_{2}\end{array}\right)$ with $\theta_{1} \in L^{s}(\Omega, d x)$ and $\theta_{2} \in L^{s}(\Gamma, d S)$, and there need not be any connection between $\theta_{1}$ and $\theta_{2}$.

Denote

$$
\begin{aligned}
v= & \gamma u, \\
p^{\prime}= & \frac{p}{p-1}, \\
\Omega_{T}= & \Omega \times(\tau, T), \\
\Gamma_{T}= & \Gamma \times(\tau, T), \\
V= & \left(L^{p}\left(\tau, T ; W^{1, p}(\Omega)\right) \cap L^{2}\left(\Omega_{T}\right) \cap L^{q}\left(\Omega_{T}\right)\right) \\
& \times\left(L^{p}\left(\tau, T ; W^{1-\frac{1}{p}, p}(\Gamma)\right) \times L^{2}\left(\Gamma_{T}\right) \cap L^{q}\left(\Gamma_{T}\right)\right), \\
V^{*}= & \left(L^{p^{\prime}}\left(\tau, T ;\left(W^{1, p}(\Omega)\right)^{*}\right)+L^{2}\left(\Omega_{T}\right)+L^{q^{\prime}}\left(\Omega_{T}\right)\right) \\
& \left.\times\left(L^{p^{\prime}}\left(\tau, T ;\left(W^{1-\frac{1}{p}, p}(\Gamma)\right)\right)^{*}\right)+L^{2}\left(\Gamma_{T}\right)+L^{q^{\prime}}\left(\Gamma_{T}\right)\right)
\end{aligned}
$$

and let the operator $A: L^{p}\left(\tau, T ; W^{1, p}(\Omega)\right) \rightarrow\left(L^{p}\left(\tau, T ; W^{1, p}(\Omega)\right)\right)^{*}$ be defined as follows:

$$
\langle A(u), v\rangle=\int_{\Omega_{T}}|\nabla u|^{p-2} \nabla u \cdot \nabla v+|u|^{p-2} u v .
$$

Next, we recall briefly some lemmas used to prove the well-posedness of the solutions and the existence of the uniform (w.r.t. $\sigma \in \Sigma$ ) attractors for (1)-(3) under some assumptions on $f$.

Lemma 2.1 [22] Let $\mathcal{O}$ be a bounded domain in $\mathbb{R}^{n}$ and $\left\{g_{n}\right\}_{n=1}^{\infty} \subset L^{q}(\mathcal{O})$, let $1<q<\infty$ be given. Assume that $\left\|g_{n}\right\|_{L^{q}(\mathcal{O})} \leq C$, where $C$ is independent of $n, g_{n} \rightarrow g$, as $n \rightarrow \infty$, almost everywhere in $\mathcal{O}$, and $g \in L^{q}(\mathcal{O})$. Then $g_{n} \rightarrow g$, as $n \rightarrow \infty$ weakly in $L^{q}(\mathcal{O})$. 
Lemma 2.2 [13] Let $x, y \in \mathbb{R}^{n}$ and $\langle\cdot, \cdot\rangle$ be the standard scalar product in $\mathbb{R}^{n}$. Then, for any $p \geq 2$, there exist two positive constants $C_{1}, C_{2}$, which depend on $p$, such that

$$
\begin{aligned}
& \left\langle|x|^{p-2} x-|y|^{p-2} y, x-y\left|\geq C_{1}\right| x-\left.y\right|^{p},\right. \\
& \left.|| x\right|^{p-2} x-|y|^{p-2} y\left|\leq C_{2}(|x|+|y|)^{p-2}\right| x-y \mid .
\end{aligned}
$$

Lemma 2.3 [23] Let $1 \leq p<\infty$ and $\Omega$ be a bounded subset of $\mathbb{R}^{n}$ with smooth boundary $\Gamma$. Then the inclusion

$$
W^{1, p}(\Omega) \hookrightarrow \hookrightarrow L^{r}(\Gamma)
$$

is compact for any $r \in\left[1, p^{*}\right)$, where

$$
p^{*}=\left\{\begin{array}{l}
\frac{(n-1) p}{n-p}, \quad p<n \\
\infty, \quad p=n
\end{array}\right.
$$

Lemma 2.4 [24] Let $A$ be defined in (7) and $X=L^{p}\left(\tau, T ; W^{1, p}(\Omega)\right)$. Then, for any $u, v \in X$, one has

$$
\langle A(u)-A(v), u-v\rangle \geq\left(\|u\|_{X}^{p-1}-\|v\|_{X}^{p-1}\right)\left(\|u\|_{X}-\|v\|_{X}\right) .
$$

Furthermore, $\langle A(u)-A(v), u-v\rangle=0$ if and only if $u=v$ a.e. in $\Omega_{T}$.

Lemma 2.5 [25] Let $X$ be a given Banach space with dual $X^{\prime}$, and let $u$ and $g$ be two functions belonging to $L^{1}(a, b ; X)$. Then the following three conditions are equivalent:

(i) $u$ is almost everywhere equal to a primitive function of $g$, i.e.,

$$
u(t)=\zeta+\int_{a}^{t} g(s) d s
$$

for almost every $t \in[a, b]$;

(ii) For each test function $\phi \in \mathbb{D}(a, b)$,

$$
\int_{a}^{b} u(t) \phi^{\prime}(t) d t=-\int_{a}^{b} g(t) \phi(t) d t \quad\left(\phi^{\prime}(t)=\frac{d \phi}{d t}\right)
$$

(iii) For each $\eta \in X^{\prime}$,

$$
\frac{d}{d t}\langle u, \eta\rangle=\langle g, \eta\rangle
$$

in the scalar distribution sense on $(a, b)$.

If (i)-(iii) are satisfied, $u$ is almost everywhere equal to a continuous function from $[a, b]$ into $X$. 


\section{The well-posedness of solutions}

In what follows, we assume that $u_{0} \in L^{2}(\bar{\Omega}, d \mu)$ is given.

Definition 3.1 A function $u(x, t)$ is called a weak solution of $(1)-(3)$ on $(\tau, T)$ if

$$
\begin{aligned}
& (u, v) \in V, \quad\left(\frac{\partial u}{\partial t}, \frac{\partial v}{\partial t}\right) \in V^{*}, \\
& \left.u\right|_{t=\tau}=u_{0} \quad \text { a.e. in } \bar{\Omega}
\end{aligned}
$$

and

$$
\begin{aligned}
& \int_{\Omega_{T}}\left(u_{t} \xi+|\nabla u|^{p-2} \nabla u \nabla \xi+|u|^{p-2} u \xi+f(u) \xi\right)+\int_{\Gamma_{T}}\left(v_{t} \xi+f(v) \xi\right) \\
& \quad=\int_{\Omega_{T}} g(x, t) \xi
\end{aligned}
$$

for all test functions $\xi \in V$.

Theorem 3.1 Let $\Omega$ be a bounded domain in $\mathbb{R}^{n}(n \geq 3)$. Assume that $f$ satisfies $\left(\mathrm{H}_{1}\right)$, $g: \Omega \times \mathbb{R} \rightarrow \mathbb{R}$ is locally Lipschitz continuous and $g \in L_{\text {loc }}^{2}\left(\mathbb{R}, L^{2}(\Omega)\right)$. Then, for any $\tau \in \mathbb{R}$, any initial data $u_{0} \in L^{2}(\bar{\Omega}, d \mu)$ and any $T>\tau$, there exists a unique weak solution $u(x, t)$ of (1)-(3), and the mapping

$$
\left(u_{0}, \gamma u_{0}\right) \rightarrow(u(t), v(t))
$$

is continuous on $L^{2}(\bar{\Omega}, d \mu)$.

Proof We first prove the existence of solutions for (1)-(5) by the Faedo-Galerkin method (see [25]).

Consider the approximating solution $u_{n}(t)$ in the form

$$
\begin{aligned}
& u_{n}(t)=\sum_{i=1}^{n} u_{n_{i}}(t) e_{i}, \\
& v_{n}(t)=\sum_{i=1}^{n} u_{n_{i}}(t) \gamma e_{i},
\end{aligned}
$$

where $\left\{\left(e_{j}, \gamma e_{j}\right)\right\}_{j=1}^{\infty}$ is an orthogonal basis of $L^{2}(\bar{\Omega}, d \mu)$, which is included in $\left(W^{1, p}(\Omega) \cap\right.$ $\left.L^{q}(\Omega)\right) \times L^{q}(\Gamma)$. We get $u_{n}$ from solving the following problem:

$$
\begin{aligned}
& \left\langle\frac{d u_{n}}{d t}, e_{k}\right\rangle+\left\langle\frac{d v_{n}}{d t}, e_{k}\right\rangle+\left\langle A\left(u_{n}\right)+\left|u_{n}\right|^{p-2} u_{n}, e_{k}\right\rangle \\
& \quad+\left\langle f\left(u_{n}\right), e_{k}\right\rangle+\left\langle f\left(v_{n}\right), e_{k}\right\rangle=\left\langle g(x, t), e_{k}\right\rangle, \\
& \left(u_{n}(\tau), e_{k}\right)=\left(u_{0}, e_{k}\right), \quad k=1, \ldots, n .
\end{aligned}
$$

Since $f$ is continuous and $g$ is locally Lipschitz continuous, using the Peano theorem, we get the local existence of $\left(u_{n}, v_{n}\right)$. Next, we establish some a priori estimates for $\left(u_{n}, v_{n}\right)$. 
We have

$$
\begin{aligned}
& \frac{1}{2} \frac{d}{d t}\left\|u_{n}(t)\right\|_{L^{2}(\Omega)}^{2}+\frac{1}{2} \frac{d}{d t}\left\|v_{n}(t)\right\|_{L^{2}(\Gamma)}^{2}+\left\|u_{n}\right\|_{1, p}^{p}+\int_{\Omega} f\left(u_{n}\right) u_{n} d x+\int_{\Gamma} f\left(v_{n}\right) v_{n} d S \\
& \quad=\int_{\Omega} g(x, t) u_{n} d x .
\end{aligned}
$$

Thanks to (5), we obtain

$$
\begin{aligned}
& \frac{1}{2} \frac{d}{d t}\left\|u_{n}(t)\right\|_{L^{2}(\Omega)}^{2}+\frac{1}{2} \frac{d}{d t}\left\|v_{n}(t)\right\|_{L^{2}(\Gamma)}^{2}+\left\|u_{n}\right\|_{1, p}^{p}+c_{1}\left\|u_{n}\right\|_{L^{q}(\Omega)}^{q}+c_{1}\left\|v_{n}\right\|_{L^{q}(\Gamma)}^{q} \\
& \quad \leq \frac{1}{2}\|g(t)\|_{L^{2}(\Omega)}^{2}+\frac{1}{2}\left\|u_{n}\right\|_{L^{2}(\Omega)}^{2}+k|\Omega|+k|\Gamma|
\end{aligned}
$$

by virtue of the following inequality (see Theorem 2.3.1 in [26]):

$$
-\mu z^{q}+\lambda z^{2} \leq C \mu^{\frac{-2}{q-2}} \lambda^{\frac{q}{q-2}} .
$$

Let $\mu=c_{1}$ and $\lambda=1$, we deduce from (10) and (12) that

$$
\begin{aligned}
& \frac{d}{d t}\left\|u_{n}(t)\right\|_{L^{2}(\Omega)}^{2}+\frac{d}{d t}\left\|v_{n}(t)\right\|_{L^{2}(\Gamma)}^{2}+2\left\|u_{n}\right\|_{1, p}^{p}+c_{1}\left\|u_{n}\right\|_{L^{q}(\Omega)}^{q}+2 c_{1}\left\|v_{n}\right\|_{L^{q}(\Gamma)}^{q} \\
& \quad \leq\|g(t)\|_{L^{2}(\Omega)}^{2}+C .
\end{aligned}
$$

Integrating (13) over $[\tau, t]$, we obtain

$$
\begin{aligned}
& \left\|u_{n}(t)\right\|_{L^{2}(\Omega)}^{2}+\left\|v_{n}(t)\right\|_{L^{2}(\Gamma)}^{2}+2 \int_{\tau}^{t}\left\|u_{n}\right\|_{1, p}^{p} d s+c_{1} \int_{\tau}^{t}\left\|u_{n}\right\|_{L^{q}(\Omega)}^{q} d s+2 c_{1} \int_{\tau}^{t}\left\|v_{n}\right\|_{L^{q}(\Gamma)}^{q} d s \\
& \leq C(T-\tau)+\int_{\tau}^{t}\|g(s)\|_{L^{2}(\Omega)}^{2} d s+\left\|u_{0}\right\|_{L^{2}(\bar{\Omega}, d \mu)}^{2}
\end{aligned}
$$

for any $t \in(\tau, T]$.

Due to (14), we get

$\left\{u_{n}\right\}$ is uniformly bounded in $L^{\infty}\left(\tau, T ; L^{2}(\Omega)\right)$,

$\left\{v_{n}\right\}$ is uniformly bounded in $L^{\infty}\left(\tau, T ; L^{2}(\Gamma)\right)$,

$\left\{u_{n}\right\}$ is uniformly bounded in $L^{p}\left(\tau, T ; W^{1, p}(\Omega)\right)$,

$\left\{u_{n}\right\}$ is uniformly bounded in $L^{q}\left(\Omega_{T}\right)$,

$\left\{v_{n}\right\}$ is uniformly bounded in $L^{q}\left(\Gamma_{T}\right)$.

Therefore, $\left\{u_{n}\right\}$ is uniformly bounded in $n$ in the $L^{p}\left(\tau, T ; W^{1, p}(\Omega)\right), L^{q}\left(\Omega_{T}\right)$, respectively, and $\left\{v_{n}\right\}$ is uniformly bounded in $n$ in the $L^{q}\left(\Gamma_{T}\right)$, and one can extract a subsequence $\left\{u_{n_{j}}\right\}$ of $\left\{u_{n}\right\}$ such that

$$
\begin{aligned}
& \left\{u_{n_{j}}\right\} \rightarrow u \text { weakly in } L^{p}\left(\tau, T ; W^{1, p}(\Omega)\right), \\
& \left\{u_{n_{j}}\right\} \rightarrow u \text { weakly in } L^{q}\left(\Omega_{T}\right), \\
& \left\{v_{n_{j}}\right\} \rightarrow v \text { weakly in } L^{q}\left(\Gamma_{T}\right) .
\end{aligned}
$$


Let $P_{n}: V \rightarrow \operatorname{span}\left\{\left(e_{j}, \gamma e_{j}\right)\right\}_{j=1}^{n}$ be a projection. For any $\phi \in V$, set $\phi_{n}=P_{n} \phi$, we have

$$
\begin{aligned}
& \left\langle\frac{d u_{n}}{d t}, \phi_{n}\right\rangle+\left\langle\frac{d v_{n}}{d t}, \phi_{n}\right\rangle+\left\langle A\left(u_{n}\right), \phi_{n}\right\rangle+\left\langle f\left(u_{n}\right), \phi_{n}\right\rangle+\left\langle f\left(v_{n}\right), \phi_{n}\right\rangle \\
& =\left\langle g(x, t), \phi_{n}\right\rangle .
\end{aligned}
$$

We perform the following estimate deduced from the Hölder inequality and the Young inequality:

$$
\begin{aligned}
\left|\left\langle A\left(u_{n}\right), \phi_{n}\right\rangle\right| & =\left.\left|\int_{\Omega_{T}}\right| \nabla u_{n}\right|^{p-2} \nabla u_{n} \cdot \nabla \phi_{n}+\left|u_{n}\right|^{p-2} u_{n} \phi_{n} d x d s \mid \\
& \leq\left\|\nabla u_{n}\right\|_{L^{p}\left(\Omega_{T}\right)}^{p-1}\left\|\nabla \phi_{n}\right\|_{L^{p}\left(\Omega_{T}\right)}+\left\|u_{n}\right\|_{L^{p}\left(\Omega_{T}\right)}^{p-1}\left\|\phi_{n}\right\|_{L^{p}\left(\Omega_{T}\right)} \\
& \leq\left\|u_{n}\right\|_{L^{p}\left(\tau, T ; W^{1, p}(\Omega)\right)}^{p-1}\left\|\phi_{n}\right\|_{L^{p}\left(\tau, T ; W^{1, p}(\Omega)\right)} .
\end{aligned}
$$

Using the boundedness of $\left\{u_{n}\right\}$ in $L^{p}\left(\tau, T ; W^{1, p}(\Omega)\right)$ again, we infer that

$$
\left\{A\left(u_{n}\right)\right\} \text { is uniformly bounded in } L^{p^{\prime}}\left(\tau, T ;\left(W^{1, p}(\Omega)\right)^{*}\right) \text {. }
$$

Since $g \in L_{\text {loc }}^{2}\left(\mathbb{R}, L^{2}(\Omega)\right), f\left(u_{n}\right) \in L^{q^{\prime}}\left(\Omega_{T}\right), f\left(v_{n}\right) \in L^{q^{\prime}}\left(\Gamma_{T}\right)$, we find

$$
\left(u_{n}^{\prime}, v_{n}^{\prime}\right) \in V^{*}
$$

Therefore we can extract a subsequence such that

$$
\begin{aligned}
& \left(u_{n}^{\prime}, v_{n}^{\prime}\right) \rightarrow\left(u^{\prime}, v^{\prime}\right) \quad \text { in } V^{*}, \\
& A\left(u_{n}\right) \rightarrow \xi \quad \text { in } L^{p^{\prime}}\left(\tau, T ;\left(W^{1, p}(\Omega)\right)^{*}\right) .
\end{aligned}
$$

By virtue of the Aubin compactness theorem, we can extract a further subsequence (still denoted by $\left.\left\{u_{n_{j}}\right\}\right)$ such that additionally

$$
\begin{aligned}
& u_{n_{j}} \rightarrow u \quad \text { in } L^{p}\left(\Omega_{T}\right), \\
& v_{n_{j}} \rightarrow v \quad \text { in } L^{p}\left(\Gamma_{T}\right) .
\end{aligned}
$$

Due to the boundedness of $\left\{u_{n}\right\}$ in $L^{q}\left(\Omega_{T}\right)$ and (5), we obtain that $\left\{f\left(u_{n}\right)\right\}$ is uniformly bounded in $L^{q^{\prime}}\left(\Omega_{T}\right)$ and hence $f\left(u_{n}\right) \rightarrow \chi$ in $L^{q^{\prime}}\left(\Omega_{T}\right)$, similarly, $f\left(v_{n}\right) \rightarrow \eta$ in $L^{q^{\prime}}\left(\Gamma_{T}\right)$. By virtue of (16)-(17), we see that $u_{n_{j}} \rightarrow u$ a.e. in $\Omega_{T}$ and $v_{n_{j}} \rightarrow v$ a.e. in $\Gamma_{T}$, then $f\left(u_{n_{j}}\right) \rightarrow f(u)$ a.e. in $\Omega_{T}$ and $f\left(v_{n_{j}}\right) \rightarrow f(v)$ a.e. in $\Gamma_{T}$. Thanks to Lemma 2.1, we know that

$$
\chi=f(u), \quad \eta=f(v)
$$

Therefore, we have

$$
\left\langle u^{\prime}, \phi\right\rangle+\left\langle v^{\prime}, \phi\right\rangle+\langle\xi, \phi\rangle+\langle f(u), \phi\rangle+\langle f(v), \phi\rangle=\langle g(x, t), \phi\rangle
$$

for any $\phi \in V$. 
In order to prove that $u$ is a weak solution of (1)-(3), it remains to show that $\xi=A(u)$. Noticing that

$$
\begin{aligned}
\left\langle A\left(u_{n}\right), u_{n}\right\rangle= & \int_{\tau}^{T}\left\|u_{n}\right\|_{1, p}^{p} d s \\
= & \frac{1}{2}\left\|u_{n}(\tau)\right\|_{L^{2}(\Omega)}^{2}-\frac{1}{2}\left\|u_{n}(T)\right\|_{L^{2}(\Omega)}^{2}+\frac{1}{2}\left\|v_{n}(\tau)\right\|_{L^{2}(\Gamma)}^{2} \\
& -\frac{1}{2}\left\|v_{n}(T)\right\|_{L^{2}(\Gamma)}^{2}-\int_{\tau}^{T} \int_{\Omega} f\left(u_{n}\right) u_{n} d x d s \\
& -\int_{\tau}^{T} \int_{\Gamma} f\left(v_{n}\right) v_{n} d S d s+\int_{\tau}^{T} \int_{\Omega} g(x, t) u_{n} d x d s,
\end{aligned}
$$

it follows from the formulation of $u_{n}(\tau)$ and $v_{n}(\tau)$ that $u_{n}(\tau) \rightarrow u_{0}$ in $L^{2}(\Omega)$ and $v_{n}(\tau) \rightarrow \theta_{0}$ in $L^{2}(\Gamma)$. Moreover, by the lower semi-continuity of $\|\cdot\|_{L^{2}(\Omega)}$ and $\|\cdot\|_{L^{2}(\Gamma)}$, we obtain

$$
\begin{aligned}
& \|u(T)\|_{L^{2}(\Omega)}^{2} \leq \liminf _{n \rightarrow \infty}\left\|u_{n}(T)\right\|_{L^{2}(\Omega)}^{2}, \\
& \|v(T)\|_{L^{2}(\Omega)}^{2} \leq \liminf _{n \rightarrow \infty}\left\|v_{n}(T)\right\|_{L^{2}(\Omega)}^{2} .
\end{aligned}
$$

Meanwhile, by the Lebesgue dominated theorem, one can check that

$$
\begin{aligned}
& \int_{\tau}^{T} \int_{\Omega} f(u) u d x d s+\int_{\tau}^{T} \int_{\Gamma} f(v) v d S d s \\
& \quad=\lim _{n \rightarrow \infty} \int_{\tau}^{T} \int_{\Omega} f\left(u_{n}\right) u_{n} d x d s+\lim _{n \rightarrow \infty} \int_{\tau}^{T} \int_{\Gamma} f\left(v_{n}\right) v_{n} d S d s, \\
& \lim _{n \rightarrow \infty} \int_{\tau}^{T} \int_{\Omega} g(x, t) u_{n} d x d s=\int_{\tau}^{T} \int_{\Omega} g(x, t) u d x d s .
\end{aligned}
$$

This fact and (20)-(21) imply

$$
\begin{aligned}
\limsup _{n \rightarrow \infty}\left\langle A\left(u_{n}\right), u_{n}\right\rangle & \\
\leq & \frac{1}{2}\|u(\tau)\|_{L^{2}(\Omega)}^{2}-\frac{1}{2}\|u(T)\|_{L^{2}(\Omega)}^{2}+\frac{1}{2}\|v(\tau)\|_{L^{2}(\Gamma)}^{2}-\frac{1}{2}\|v(T)\|_{L^{2}(\Gamma)}^{2} \\
& \quad-\int_{\tau}^{T} \int_{\Omega} f(u) u d x d s-\int_{\tau}^{T} \int_{\Gamma} f(v) v d S d s+\int_{\tau}^{T} \int_{\Omega} g(x, t) u d x d s .
\end{aligned}
$$

In view of (18), we have

$$
\begin{aligned}
\langle\xi, u\rangle= & \frac{1}{2}\|u(\tau)\|_{L^{2}(\Omega)}^{2}-\frac{1}{2}\|u(T)\|_{L^{2}(\Omega)}^{2}+\frac{1}{2}\|v(\tau)\|_{L^{2}(\Gamma)}^{2}-\frac{1}{2}\|v(T)\|_{L^{2}(\Gamma)}^{2} \\
& -\int_{\tau}^{T} \int_{\Omega} f(u) u d x d s-\int_{\tau}^{T} \int_{\Gamma} f(v) v d S d s+\int_{\tau}^{T} \int_{\Omega} g(x, t) u d x d s .
\end{aligned}
$$

This and (22) deduce

$$
\limsup _{n \rightarrow \infty}\left\langle A\left(u_{n}\right), u_{n}\right\rangle \leq\langle\xi, u\rangle .
$$


To this end, we first observe that

$$
\begin{aligned}
& \lim _{n \rightarrow \infty}\left\langle A\left(u_{n}\right)-A(u), u_{n}-u\right\rangle \\
& \quad=\lim _{n \rightarrow \infty}\left(\left\langle A\left(u_{n}\right), u_{n}\right\rangle-\left\langle A\left(u_{n}\right), u\right\rangle-\left\langle A(u), u_{n}-u\right\rangle\right) \\
& \quad \leq\langle\xi, u\rangle-\langle\xi, u\rangle=0 .
\end{aligned}
$$

On the other hand, it follows from Lemma 2.4 that

$$
\begin{aligned}
& \left\langle A\left(u_{n}\right)-A(u), u_{n}-u\right\rangle \\
& \quad \geq\left(\left\|u_{n}\right\|_{L^{p}\left(\tau, T ; W^{1, p}(\Omega)\right)}^{p-1}-\|u\|_{L^{p}\left(\tau, T ; W^{1, p}(\Omega)\right)}^{p-1}\right)\left(\left\|u_{n}\right\|_{L^{p}\left(\tau, T ; W^{1, p}(\Omega)\right)}-\|u\|_{L^{p}\left(\tau, T ; W^{1, p}(\Omega)\right)}\right) \geq 0 .
\end{aligned}
$$

Hence

$$
\left\|u_{n}\right\|_{L^{p}\left(\tau, T ; W^{1, p}(\Omega)\right)} \rightarrow\|u\|_{L^{p}\left(\tau, T ; W^{1, p}(\Omega)\right)}, \quad \text { as } n \rightarrow \infty .
$$

Combining (24) with $u_{n} \rightarrow u$ in $L^{p}\left(\tau, T ; W^{1, p}(\Omega)\right)$, we obtain

$$
u_{n} \rightarrow u \quad \text { in } L^{p}\left(\tau, T ; W^{1, p}(\Omega)\right)
$$

Therefore, from Lemma 2.2, the Hölder inequality and the Young inequality, we deduce that for any $\phi \in L^{p}\left(\tau, T ; W^{1, p}(\Omega)\right)$,

$$
\begin{aligned}
& \mid\left\langle A\left(u_{n}\right)-A(u), \phi||\right. \\
& =\left|\int_{\Omega_{T}}\left(\left|\nabla u_{n}\right|^{p-2} \nabla u_{n}-|\nabla u|^{p-2} \nabla u\right) \cdot \nabla \phi+\left(\left|u_{n}\right|^{p-2} u_{n}-|u|^{p-2} u\right) \phi d x d s\right| \\
& \leq C_{2} \int_{\Omega_{T}}\left(\left|\nabla u_{n}\right|+|\nabla u|\right)^{p-2}\left|\nabla u_{n}-\nabla u\right||\nabla \phi| d x d s \\
& \quad+C_{2} \int_{\Omega_{T}}\left(\left|u_{n}\right|+|u|\right)^{p-2}\left|u_{n}-u\right||\phi| d x d s \\
& \leq C\left(\left\|u_{n}\right\|_{L^{p}\left(\tau, T ; W^{1, p}(\Omega)\right)}^{p-2}+\|u\|_{L^{p}\left(\tau, T ; W^{1, p}(\Omega)\right)}^{p-2}\right) \\
& \quad \times\left\|u_{n}-u\right\|_{L^{p}\left(\tau, T ; W^{1, p}(\Omega)\right)}\|\phi\|_{L^{p}\left(\tau, T ; W^{1, p}(\Omega)\right)},
\end{aligned}
$$

which implies that $A\left(u_{n}\right) \rightarrow A(u)$ in $\left(L^{p}\left(\tau, T ; W^{1, p}(\Omega)\right)\right)^{*}$, hence $\xi=A(u)$.

Finally, we prove the uniqueness and continuous dependence of the initial data of the solutions. Let $u^{1}, u^{2}$ be two solutions of (1)-(5) with the initial data $u_{0}^{1}, u_{0}^{2}$, respectively. Let $w=u^{1}-u^{2}$. Taking the inner product of the equation with $w$, we deduce that

$$
\begin{aligned}
& \frac{1}{2} \frac{d}{d t}\|w(t)\|_{L^{2}(\Omega)}^{2}+\frac{1}{2} \frac{d}{d t}\|w(t)\|_{L^{2}(\Gamma)}^{2}+\int_{\Omega}\left(\left|u^{1}\right|^{p-2} u^{1}-\left|u^{2}\right|^{p-2} u^{2}, u^{1}-u^{2}\right) d x \\
& \quad+\int_{\Omega}\left(\left|\nabla u^{1}\right|^{p-2} \nabla u^{1}-\left|\nabla u^{2}\right|^{p-2} \nabla u^{2}, \nabla u^{1}-\nabla u^{2}\right) d x \\
& \quad+\int_{\Omega}\left(f\left(u^{1}\right)-f\left(u^{2}\right), u^{1}-u^{2}\right) d x+\int_{\Gamma}\left(f\left(v^{1}\right)-f\left(v^{2}\right), v^{1}-v^{2}\right) d S=0 .
\end{aligned}
$$


By virtue of (4) and Lemma 2.2, we obtain

$$
\begin{aligned}
& \frac{1}{2} \frac{d}{d t}\|w(t)\|_{L^{2}(\Omega)}^{2}+\frac{1}{2} \frac{d}{d t}\|w(t)\|_{L^{2}(\Gamma)}^{2} \\
& \quad \leq l\|w(t)\|_{L^{2}(\Omega)}^{2}+l\|w(t)\|_{L^{2}(\Gamma)}^{2}
\end{aligned}
$$

which implies that

$$
\begin{aligned}
& \|w(t)\|_{L^{2}(\Omega)}^{2}+\|w(t)\|_{L^{2}(\Gamma)}^{2} \\
& \quad \leq \exp (2 l(t-\tau))\left(\|w(\tau)\|_{L^{2}(\Omega)}^{2}+\|w(\tau)\|_{L^{2}(\Gamma)}^{2}\right) .
\end{aligned}
$$

Therefore, $u^{1}(x, t)=u^{2}(x, t)$ a.e. in $\overline{\Omega_{T}}$ if $u_{0}^{1}(x)=u_{0}^{2}(x)$ in $\bar{\Omega}$, and $u(x, t)$ is continuously dependent on the initial data.

Since

$$
\begin{aligned}
& (u(t), v(t)) \in V, \\
& \left(u_{t}(t), v_{t}(t)\right) \in V^{*},
\end{aligned}
$$

by use of Lemma 2.5, we know that

$$
(u(t), v(t)) \in C\left([\tau, T] ; L^{2}(\bar{\Omega}, d \mu)\right) .
$$

Therefore, $(u(\tau), v(\tau)) \in L^{2}(\bar{\Omega}, d \mu)$ is meaningful.

By Theorem 3.1, we can define a family of continuous processes $\{U(t, \tau):-\infty<\tau \leq t<$ $\infty\}$ in $L^{2}(\bar{\Omega}, d \mu)$ as follows: For all $t \geq \tau$,

$$
U(t, \tau)\left(u_{0}, \gamma u_{0}\right)=(u(t), v(t)):=\left(u\left(t ; \tau,\left(u_{0}, \gamma u_{0}\right)\right), v\left(t ; \tau,\left(u_{0}, \gamma u_{0}\right)\right)\right)
$$

where $u(t)$ is the solution of (1)-(5) with initial data $(u(\tau), v(\tau))=\left(u_{0}, \gamma u_{0}\right) \in L^{2}(\bar{\Omega}, d \mu)$. That is, a family of mappings $U(t, \tau): L^{2}(\bar{\Omega}, d \mu) \rightarrow L^{2}(\bar{\Omega}, d \mu)$ satisfies

$$
\begin{aligned}
& U(\tau, \tau)=i d \quad \text { (identity), } \\
& U(t, \tau)=U(t, r) U(r, \tau) \quad \text { for all } \tau \leq r \leq t
\end{aligned}
$$

\section{Existence of uniform attractors}

In this section, we prove the existence of uniform attractors for (1)-(3).

\subsection{Abstract results}

In this subsection, let $\Sigma$ be a parameter set, let $X, Y$ be two Banach spaces, $Y \subset X$ continuously. $\left\{U_{\sigma}(t, \tau)\right\}_{\sigma \in \Sigma}$ is a family of processes in a Banach space $X$. Denote by $B(X)$ the set of all bounded subsets of $X$ and $\mathbb{R}_{\tau}=[\tau,+\infty)$. In the following, we give some basic definitions and some abstract results about the existence of bi-space uniform (with respect to (w.r.t.) $\sigma \in \Sigma$ ) attractors. 
Definition 4.1 [5,27] A set $B_{0} \subset B(Y)$ is called to be $(X, Y)$-uniformly (w.r.t. $\sigma \in \Sigma$ ) absorbing for $\left\{U_{\sigma}(t, \tau)\right\}_{\sigma \in \Sigma}$ if for any $\tau \in \mathbb{R}$ and any bounded subset $B \subset X$, there exists a positive constant $t_{0}=t_{0}(\tau, B) \geq \tau$ such that

$$
\bigcup_{\sigma \in \Sigma} U_{\sigma}(t, \tau) B \subset B_{0}
$$

for any $t \geq t_{0}$.

A set $P \subset Y$ is said to be $(X, Y)$-uniformly (w.r.t. $\sigma \in \Sigma)$ attracting for the family of processes $\left\{U_{\sigma}(t, \tau)\right\}_{\sigma \in \Sigma}$, if

$$
\sup _{\sigma \in \Sigma} \operatorname{dist}_{Y}\left(U_{\sigma}(t+\tau, \tau) B, P\right) \rightarrow 0 \quad(t \rightarrow \infty)
$$

for an arbitrary fixed $\tau \in \mathbb{R}$ and any bounded set $B \subset X$.

Definition 4.2 [5] A closed set $A_{\Sigma} \subset Y$ is said to be an $(X, Y)$-uniform (w.r.t. $\sigma \in \Sigma$ ) attractor for the family of processes $\left\{U_{\sigma}(t, \tau)\right\}_{\sigma \in \Sigma}$ if it is $(X, Y)$-uniformly (w.r.t. $\sigma \in \Sigma$ ) attracting and it is contained in any closed $(X, Y)$-uniformly (w.r.t. $\sigma \in \Sigma$ ) attracting set $A^{\prime}$ for the family of processes $\left\{U_{\sigma}(t, \tau)\right\}_{\sigma \in \Sigma}: A_{\Sigma} \subset A^{\prime}$.

Definition 4.3 [5] Define the uniform (w.r.t. $\sigma \in \Sigma$ ) $\omega$-limit set of $B$ by $\omega_{\tau, \Sigma}(B)=$ $\bigcap_{t \geq \tau} \bar{\bigcup}_{\sigma \in \Sigma} \bigcup_{s \geq t} U_{\sigma}(s, \tau) B$. This can be characterized by the following: $y \in \omega_{\tau, \Sigma}(B)$ if and only if there are sequences $\left\{x_{n}\right\} \subset B,\left\{\sigma_{n}\right\} \subset \Sigma,\left\{t_{n}\right\} \subset \mathbb{R}_{\tau}, t_{n} \rightarrow \infty$ such that $U_{\sigma_{n}}\left(t_{n}, \tau\right) x_{n} \rightarrow$ $y(n \rightarrow \infty)$.

Definition 4.4 [5] A family of processes $\left\{U_{\sigma}(t, \tau)\right\}_{\sigma \in \Sigma}$ possessing a compact $(X, Y)$ uniformly (w.r.t. $\sigma \in \Sigma$ ) absorbing set is called $(X, Y)$-uniformly compact. A family of processes $\left\{U_{\sigma}(t, \tau)\right\}_{\sigma \in \Sigma}$ is called $(X, Y)$-uniformly asymptotically compact if it possesses a compact $(X, Y)$-uniformly (w.r.t. $\sigma \in \Sigma$ ) attracting set, i.e., for any bounded subset $B \subset X$ and any sequences $\left\{\tau_{n}\right\} \subset \mathbb{R}, t_{n} \rightarrow+\infty$ as $n \rightarrow+\infty$ and $\left\{x_{n}\right\} \subset B,\left\{U\left(t_{n}+\tau_{n}, \tau_{n}\right) x_{n}\right\}_{n=1}^{\infty}$ is precompact in $Y$.

Lemma 4.1 [20] If a family of processes $\left\{U_{\sigma}(t, \tau)\right\}_{\sigma \in \Sigma}$ is $(X, Y)$-uniformly asymptotically compact, then for any $\tau \in \mathbb{R}, B \subset B(X)$,

(i) for any sequences $\left\{x_{n}\right\} \subset B,\left\{\sigma_{n}\right\} \subset \Sigma,\left\{t_{n}\right\} \subset \mathbb{R}_{\tau}, t_{n} \rightarrow \infty$ as $n \rightarrow \infty$, there is a convergent subsequence of $\left\{U_{\sigma_{n}}\left(t_{n}, \tau\right) x_{n}\right\}$ in $Y$,

(ii) $\omega_{\tau, \Sigma}(B)$ is nonempty and compact in $Y$,

(iii) $\omega_{\tau, \Sigma}(B)=\omega_{0, \Sigma}(B)$,

(iv) $\lim _{t \rightarrow \infty}\left(\sup _{\sigma \in \Sigma} \operatorname{dist}_{Y}\left(U_{\sigma}(t, \tau) B, \omega_{\tau, \Sigma}(B)\right)\right)=0$,

(v) if $A$ is a closed set and $(X, Y)$-uniformly (w.r.t. $\sigma \in \Sigma$ ) attracting $B$, then $\omega_{\tau, \Sigma}(B) \subset A$.

Assumption 1 Let $\{T(h) \mid h \geq 0\}$ be a family of operators acting on $\Sigma$ and satisfying:

(i) $T(h) \Sigma=\Sigma, \forall h \in \mathbb{R}^{+}$,

(ii) translation identity:

$$
U_{\sigma}(t+h, \tau+h)=U_{T(h) \sigma}(t, \tau), \quad \forall \sigma \in \Sigma, t \geq \tau, \tau \in \mathbb{R}, h \geq 0 .
$$


Definition 4.5 [5] The kernel $\mathcal{K}$ of the process $\left\{U_{\sigma}(t, \tau)\right\}$ acting on $X$ consists of all bounded complete trajectories of the process $\left\{U_{\sigma}(t, \tau)\right\}$ :

$$
\mathcal{K}=\left\{u(\cdot) \mid U(t, \tau) u(\tau)=u(t), \operatorname{dist}(u(t), u(0)) \leq C_{u}, \forall t \geq \tau, \tau \in \mathbb{R}\right\} .
$$

The set $\mathcal{K}(s)=\{u(s) \mid u(\cdot) \in \mathcal{K}\}$ is said to be kernel section at time $t=s, s \in \mathbb{R}$.

Definition 4.6 [5] A family of processes $\left\{U_{\sigma}(t, \tau)\right\}_{\sigma \in \Sigma}$ is said to be $(X \times \Sigma, Y)$-weakly continuous if for any fixed $t \geq \tau, \tau \in \mathbb{R}$, the mapping $(u, \sigma) \rightarrow U_{\sigma}(t, \tau) u$ is weakly continuous from $X \times \Sigma$ to $Y$.

Assumption 2 Let $\Sigma$ be a weakly compact set and $\left\{U_{\sigma}(t, \tau)\right\}_{\sigma \in \Sigma}$ be $(X \times \Sigma, Y)$-weakly continuous.

Lemma 4.2 [20] Under Assumptions 1 and 2 with $\{T(h)\}_{h \geq 0}$, which is a weakly continuous semigroup, if $\left\{U_{\sigma}(t, \tau)\right\}_{\sigma \in \Sigma}$ acting on $X$ is $(X, Y)$-uniformly (w.r.t. $\left.\sigma \in \Sigma\right)$ asymptotically compact, then it possesses an $(X, Y)$-uniform (w.r.t. $\sigma \in \Sigma)$ attractor $\mathcal{A}_{\Sigma}$, which is compact in $Y$ and attracts all the bounded subsets of $X$ in the topology of $Y$.

Moreover,

$$
\mathcal{A}_{\Sigma}=\omega_{\tau, \Sigma}\left(B_{0}\right)=\bigcup_{\sigma \in \Sigma} \mathcal{K}_{\sigma}(s), \quad \forall s \in \mathbb{R}
$$

where $B_{0}$ is a bounded neighborhood of the compact $(X, Y)$-uniformly attracting set in $Y$; i.e., $B_{0}$ is a bounded $(X, Y)$-uniformly (w.r.t. $\left.\sigma \in \Sigma\right)$ absorbing set of $\left\{U_{\sigma}(t, \tau)\right\}_{\sigma \in \Sigma}$. $\mathcal{K}_{\sigma}(s)$ is the section at $t=s$ of kernel $\mathcal{K}_{\sigma}$ of the process $\left\{U_{\sigma}(t, \tau)\right\}$ with symbol $\sigma \in \Sigma$. Furthermore, $\mathcal{K}_{\sigma}$ is nonempty for all $\sigma \in \Sigma$.

From the ideas of $[4,20,28]$, we give the following results, which are very useful for the existence of a uniform attractor in $L^{p}(\bar{\Omega}, d \mu)$.

Lemma 4.3 [20] Let $\left\{U_{\sigma}(t, \tau)\right\}_{\sigma \in \Sigma}$ be a family of processes on $L^{p}(\Omega)(p \geq 1)$ and suppose $\left\{U_{\sigma}(t, \tau)\right\}_{\sigma \in \Sigma}$ has a bounded $\left(L^{p}(\Omega), L^{p}(\Omega)\right)$-uniformly (w.r.t. $\left.\sigma \in \Sigma\right)$ absorbing set in $L^{p}(\Omega)$. Then, for any $\epsilon>0, \tau \in \mathbb{R}$ and any bounded subset $B \in L^{p}(\Omega)$, there exist two positive constants $T=T(B, \tau)$ and $M=M(\epsilon)$ such that

$$
m\left(\Omega\left(\left|U_{\sigma}(t, \tau) u_{\tau}\right| \geq M\right)\right) \leq \epsilon
$$

for any $u_{\tau} \in B, t \geq T, \sigma \in \Sigma$.

Lemma 4.4 $[4,28]$ Let a family of processes $\left\{U_{\sigma}(t, \tau)\right\}_{\sigma \in \Sigma}$ be $\left(L^{p}(\Omega), L^{p}(\Omega)\right)$-uniformly (w.r.t. $\sigma \in \Sigma$ ) asymptotically compact, then $\left\{U_{\sigma}(t, \tau)\right\}_{\sigma \in \Sigma}$ is $\left(L^{p}(\Omega), L^{q}(\Omega)\right)$-uniformly asymptotically compact for $p \leq q<\infty$, if

(i) $\left\{U_{\sigma}(t, \tau)\right\}_{\sigma \in \Sigma}$ has a bounded $\left(L^{p}(\Omega), L^{q}(\Omega)\right)$-uniformly (w.r.t. $\left.\sigma \in \Sigma\right)$ absorbing set $B_{0}$, 
(ii) for any $\epsilon>0, \tau \in \mathbb{R}$ and any bounded subset $B \subset L^{p}(\Omega)$, there exist two positive constants $M=M(\epsilon, B)$ and $T=T(\epsilon, B, \tau)$ such that

$$
\int_{\Omega\left(\left|U_{\sigma}(t, \tau) u_{\tau}\right| \geq M\right)}\left|U_{\sigma}(t, \tau) u_{\tau}\right|^{q} \leq \epsilon \quad \text { for all } u_{\tau} \in B, t \geq T, \sigma \in \Sigma
$$

From Theorem 3.1, we know that the problem (1)-(5) generates a process $\left\{U_{\sigma}(t, \tau)\right\}_{\sigma \in \Sigma}$ acting in $L^{2}(\bar{\Omega}, d \mu)$ and the time symbol is $\sigma(s)=g(x, s)$. We denote by $L_{\text {loc }}^{2, w}\left(\mathbb{R} ; L^{2}(\Omega)\right)$ the space $L_{\text {loc }}^{2}\left(\mathbb{R} ; L^{2}(\Omega)\right)$ endowed with a locally weak convergence topology. Let $\mathcal{H}_{w}(g)$ be the hull of $g$ in $L_{\text {loc }}^{2, w}\left(\mathbb{R} ; L^{2}(\Omega)\right)$, i.e., the closure of the set $\{g(s+h) \mid h \in \mathbb{R}\}$ in $L_{\text {loc }}^{2, w}\left(\mathbb{R} ; L^{2}(\Omega)\right)$ and $g(x, s) \in L_{b}^{2}\left(\mathbb{R} ; L^{2}(\Omega)\right)$.

Lemma 4.5 [5] If $\mathcal{E}$ is reflective separable and $\phi \in L_{b}^{2}(\mathbb{R} ; \mathcal{E})$, then

(i) for all $\phi_{1} \in \mathcal{H}_{w}(\phi),\left\|\phi_{1}\right\|_{L_{b}^{2}}^{2} \leq\|\phi\|_{L_{b}^{2}}^{2}$,

(ii) the translation group $\{T(h)\}$ is weakly continuous on $\mathcal{H}_{w}(\phi)$,

(iii) $T(h) \mathcal{H}_{w}(\phi)=\mathcal{H}_{w}(\phi)$ for $h \geq 0$,

(iv) $\mathcal{H}_{w}(\phi)$ is weakly compact.

Due to Lemma $4.5, \mathcal{H}_{w}(g)$ is weakly compact and the translation semigroup $\{T(h) \mid h \in$ $\left.\mathbb{R}^{+}\right\}$satisfies that $T(h) \mathcal{H}_{w}(g)=\mathcal{H}_{w}(g)$ and is weakly continuous on $\mathcal{H}_{w}(g)$. Because of the uniqueness of solution, the following translation identity holds:

$$
U_{\sigma}(t+h, \tau+h)=U_{T(h) \sigma}(t, \tau) \quad \forall \sigma \in \mathcal{H}_{w}(g), t \geq \tau, \tau \in \mathbb{R}, h \geq 0
$$

Theorem 4.1 The family of processes $\left\{U_{\sigma}(t, \tau)\right\}_{\sigma \in \mathcal{H}_{w}(g)}$ corresponding to problem (1)-(5) is $\left(L^{2}(\bar{\Omega}, d \mu) \times \mathcal{H}_{w}(g), L^{2}(\bar{\Omega}, d \mu)\right)$-weakly continuous and $\left(L^{2}(\bar{\Omega}, d \mu) \times \mathcal{H}_{w}(g),\left(L^{q}(\Omega) \cap\right.\right.$ $\left.\left.W^{1, p}(\Omega)\right) \times L^{q}(\Gamma)\right)$-weakly continuous.

Proof For any fixed $t_{1}$ and $\tau, t_{1} \geq \tau, \tau \in \mathbb{R}$, let $u_{\tau_{n}} \rightarrow u_{\tau}(n \rightarrow \infty)$ weakly in $L^{2}(\bar{\Omega}, d \mu)$ and $\sigma_{n} \rightarrow \sigma_{0}$ weakly in $\mathcal{H}_{w}(g)$ as $n \rightarrow \infty$, denote by $u_{n}(t)=U_{\sigma_{n}}(t, \tau) u_{\tau_{n}}$. The same estimates for $u_{n} \in E_{n}=\operatorname{span}\left\{\left(e_{i}, \gamma e_{i}\right)\right\}_{i=1}^{n}$ given in the Galerkin approximations (in Section 3) are valid for the $u_{n}(t)$ here. Therefore, for some subsequence $\{m\} \subset\{n\}$ and $u(t)$ such that for any $t_{1}, \tau \leq t_{1} \leq t,\left(u_{m}\left(t_{1}\right), v_{m}\left(t_{1}\right)\right) \rightarrow\left(u\left(t_{1}\right), v\left(t_{1}\right)\right)$ weakly in $L^{2}(\bar{\Omega}, d \mu)$ and $\left(L^{q}(\Omega) \cap W^{1, p}(\Omega)\right) \times L^{q}(\Gamma)$. And the sequence $\left\{\left(u_{m}(s), v_{m}(s)\right)\right\}, \tau \leq s \leq t$ is bounded in $L^{\infty}\left(\tau, t ; L^{2}(\bar{\Omega}, d \mu) \cap\left(\left(L^{p}\left(\tau, t ; W^{1, p}(\Omega)\right) \cap L^{q}\left(\tau, t ; L^{q}(\Omega)\right)\right) \times L^{q}\left(\tau, t ; L^{q}(\Gamma)\right)\right)\right.$. Denote by $\xi(s)$, $\chi(s)$ and $\eta(s)$ the weak limits of $A\left(u_{m}\right)(s), f\left(u_{m}(s)\right)$ and $f\left(v_{m}(s)\right)$ in $L^{p^{\prime}}\left(\tau, t ;\left(W^{1, p}(\Omega)\right)^{*}\right)$, $L^{q^{\prime}}\left(\tau, t ; L^{q^{\prime}}(\Omega)\right)$ and $L^{q^{\prime}}\left(\tau, t ; L^{q^{\prime}}(\Gamma)\right)$, respectively. So, we get the following equation for $u(s)$ :

$$
\left\langle\partial_{t} u, \phi\right\rangle+\left\langle\partial_{t} v, \gamma \phi\right\rangle+\left\langle\eta_{0}+\eta_{1}, \phi\right\rangle+\left\langle\eta_{2}, \gamma \phi\right\rangle=\left\langle\sigma_{0}, \phi\right\rangle
$$

for any $\phi \in V$.

By the same method as the proof of Theorem 3.1, we know that $\eta_{0}=A(u), \eta_{1}=f(u)$ and $\eta_{2}=f(v)$, which means that $(u(s), v(s))$ in $V$ is the weak solution of (1)-(5) with the initial condition $u_{\tau}$. Due to the uniqueness of the solution, we state that $U_{\sigma_{m}}\left(t_{1}, \tau\right)\left(u_{\tau_{m}}, \gamma u_{\tau_{m}}\right) \rightarrow$ $U_{\sigma_{0}}\left(t_{1}, \tau\right)\left(u_{\tau}, \gamma u_{\tau}\right)$ weakly in $L^{2}(\bar{\Omega}, d \mu)$ and $\left(L^{q}(\Omega) \cap W^{1, p}(\Omega)\right) \times L^{q}(\Gamma)$. For any other subsequence, $\left\{u_{\tau_{m^{\prime}}}\right\}$ and $\left\{\sigma_{m^{\prime}}\right\}$ satisfy $u_{\tau_{m^{\prime}}} \rightarrow u_{\tau}$ weakly in $L^{2}(\bar{\Omega}, d \mu)$ and $\sigma_{m^{\prime}} \rightarrow \sigma_{0}$, by the same process, we obtain the analogous relation $U_{\sigma_{m^{\prime}}}\left(t_{1}, \tau\right) u_{\tau_{m^{\prime}}} \rightarrow U_{\sigma_{0}}\left(t_{1}, \tau\right) u_{\tau}$ weakly in 
$L^{2}(\bar{\Omega}, d \mu)$ and $\left(L^{q}(\Omega) \cap W^{1, p}(\Omega)\right) \times L^{q}(\Gamma)$ holds. Then it can be easily seen that for any weakly convergent initial sequence $\left\{u_{\tau_{n}}\right\} \in L^{2}(\bar{\Omega}, d \mu)$ and weakly convergent sequence $\left\{\sigma_{n}\right\} \in \mathcal{H}_{w}(g)$, we have $U_{\sigma_{n}}\left(t_{1}, \tau\right) u_{\tau_{n}} \rightarrow U_{\sigma_{0}}\left(t_{1}, \tau\right) u_{\tau}$ weakly in $L^{2}(\bar{\Omega}, d \mu)$ and $\left(L^{q}(\Omega) \cap\right.$ $\left.W^{1, p}(\Omega)\right) \times L^{q}(\Gamma)$.

Lemma 4.6 [25] (The uniform Gronwall lemma) Let $x(t), a(t), b(t)$ be three positive locally integrable functions on $\left[t_{0}, \infty\right)$, and for some $r>0$ and all $t \geq t_{0}, x(t), a(t), b(t)$ satisfy the following inequalities:

$$
x^{\prime}(t) \leq a(t) x(t)+b(t)
$$

and

$$
\int_{t}^{t+r} x(\tau) d \tau \leq R, \quad \int_{t}^{t+r} a(\tau) d \tau \leq A, \quad \int_{t}^{t+r} b(\tau) d \tau \leq B
$$

where $R, A, B$ are three positive constants. Then

$$
x(t) \leq\left(\frac{R}{r}+B\right) e^{A}
$$

for all $t \geq t_{0}+r$.

\subsection{The existence of uniformly absorbing sets}

In this subsection, we prove the existence of uniformly (w.r.t. $\sigma \in \Sigma$ ) absorbing sets for the process $\left\{U_{\sigma}(t, \tau)\right\}_{\sigma \in \Sigma}$ corresponding to (1)-(5).

Theorem 4.2 Assume that $f$ and $g$ satisfy $\left(\mathrm{H}_{1}\right)-\left(\mathrm{H}_{2}\right)$. Then the family of processes $\left\{U_{\sigma}(t, \tau)\right\}_{\sigma \in \mathcal{H}_{w}(g)}$ corresponding to problem (1)-(5) has a bounded $\left(L^{2}(\bar{\Omega}, d \mu), L^{2}(\bar{\Omega}, d \mu)\right)-$ and $\left(L^{2}(\bar{\Omega}, d \mu),\left(L^{q}(\Omega) \cap W^{1, p}(\Omega)\right) \times L^{q}(\Gamma)\right)$-uniformly (w.r.t. $\left.\sigma \in \mathcal{H}_{w}(g)\right)$ absorbing set. That is, for any bounded subset $B$ of $L^{2}(\bar{\Omega}, d \mu)$ and any $\tau \in \mathbb{R}$, there exist $\tau_{1}=\tau_{1}(\tau, B)$, $\tau_{2}=\tau_{2}(\tau, B) \geq \tau$ and two positive constants $\rho_{1}, \rho_{2}$ such that

$$
\|u(t)\|_{L^{2}(\Omega)}^{2}+\|v(t)\|_{L^{2}(\Gamma)}^{2} \leq 3 \rho_{1}
$$

for any $t \geq \tau_{1}$ and

$$
\|u(t)\|_{W^{1, p}(\Omega)}^{p}+\|u(t)\|_{L^{q}(\Omega)}^{q}+\|v(t)\|_{L^{q}(\Gamma)}^{q} \leq C \rho_{2}
$$

for any $t \geq \tau_{2}$, where $\tau_{1}, \tau_{2}, \rho_{1}$, and $\rho_{2}$ are specified in (33), (41), (32) and (40), respectively.

Proof Taking the inner product of (1) with $u$, we deduce that

$$
\begin{aligned}
& \frac{1}{2} \frac{d}{d t}\left(\|u\|_{L^{2}(\Omega)}^{2}+\|v\|_{L^{2}(\Gamma)}^{2}\right)+\|u\|_{W^{1, p}}^{p}+\int_{\Omega} f(u) u d x+\int_{\Gamma} f(v) v d S \\
& \quad=\int_{\Omega} \sigma(t) u d x .
\end{aligned}
$$


By virtue of (5), the Hölder inequality and the Young inequality, we obtain

$$
\begin{aligned}
& \frac{1}{2} \frac{d}{d t}\left(\|u\|_{L^{2}(\Omega)}^{2}+\|v\|_{L^{2}(\Gamma)}^{2}\right)+\|u\|_{W^{1, p}(\Omega)}^{p}+c_{1}\|u\|_{L^{q}(\Omega)}^{q}+c_{1}\|v\|_{L^{q}(\Gamma)}^{q} \\
& \quad \leq \frac{1}{2}\|\sigma(t)\|_{L^{2}(\Omega)}^{2}+\frac{1}{2}\|u\|_{L^{2}(\Omega)}^{2}+k|\Omega|+k|\Gamma| \\
& \quad \leq \frac{1}{2}\|\sigma(t)\|_{L^{2}(\Omega)}^{2}+\frac{1}{2}\|u\|_{L^{2}(\Omega)}^{2}+\frac{1}{2}\|v\|_{L^{2}(\Gamma)}^{2}+k|\Omega|+k|\Gamma| .
\end{aligned}
$$

Let $\mu=c_{1}$ and $\lambda=2$, we deduce from (12) and (29) that

$$
\begin{aligned}
& \frac{d}{d t}\|u(t)\|_{L^{2}(\Omega)}^{2}+\frac{d}{d t}\|v(t)\|_{L^{2}(\Gamma)}^{2}+2\|u\|_{1, p}^{p}+c_{1}\|u\|_{L^{q}(\Omega)}^{q} \\
& \quad+c_{1}\|v\|_{L^{q}(\Gamma)}^{q}+\|u\|_{L^{2}(\Omega)}^{2}+\|v\|_{L^{2}(\Gamma)}^{2} \\
& \leq\|\sigma(t)\|_{L^{2}(\Omega)}^{2}+C .
\end{aligned}
$$

It follows from the classical Gronwall inequality and Lemma 4.5 that

$$
\begin{aligned}
& \|u(t)\|_{L^{2}(\Omega)}^{2}+\|v(t)\|_{L^{2}(\Gamma)}^{2} \\
& \leq\left\|u_{0}\right\|_{L^{2}(\bar{\Omega}, d \mu)}^{2} e^{\tau-t}+\int_{\tau}^{t} e^{s-t}\|g(s)\|_{L^{2}(\Omega)}^{2} d s+C \\
& \leq\left\|u_{0}\right\|_{L^{2}(\bar{\Omega}, d \mu)}^{2} e^{\tau-t}+2 \sup _{r \in \mathbb{R}} \int_{r}^{r+1}\|g(s)\|_{L^{2}(\Omega)}^{2} d s+C,
\end{aligned}
$$

where we have used the following inequality:

$$
\begin{aligned}
& \int_{\tau}^{t} e^{s-t}\|g(s)\|_{L^{2}(\Omega)}^{2} d s \\
& \quad=\int_{t-1}^{t} e^{s-t}\|g(s)\|_{L^{2}(\Omega)}^{2} d s+\int_{t-2}^{t-1} e^{s-t}\|g(s)\|_{L^{2}(\Omega)}^{2} d s+\cdots \\
& \leq\left(1+e^{-1}+e^{-2}+\cdots+e^{-n}+\cdots\right) \sup _{r \in \mathbb{R}} \int_{r}^{r+1}\|g(s)\|_{L^{2}(\Omega)}^{2} d s \\
& \quad \leq \frac{1}{1-e^{-1}} \sup _{r \in \mathbb{R}} \int_{r}^{r+1}\|g(s)\|_{L^{2}(\Omega)}^{2} d s \\
& \quad \leq 2 \sup _{r \in \mathbb{R}} \int_{r}^{r+1}\|g(s)\|_{L^{2}(\Omega)}^{2} d s .
\end{aligned}
$$

From (31), we deduce that

$$
\|u(t)\|_{L^{2}(\Omega)}^{2}+\|v(t)\|_{L^{2}(\Gamma)}^{2} \leq 3 \rho_{1},
$$

where

$$
\begin{aligned}
& \rho_{1}=\sup _{r \in \mathbb{R}} \int_{r}^{r+1}\|g(s)\|_{L^{2}(\Omega)}^{2} d s+C, \\
& \tau_{1}=\tau+\max \left\{0, \ln \left(\frac{\left\|u_{0}\right\|_{L^{2}(\bar{\Omega}, d \mu)}^{2}}{\rho_{1}}\right)\right\} .
\end{aligned}
$$


Integrating (30) over $[r, r+1]$, we obtain

$$
\begin{aligned}
& c_{1} \int_{r}^{r+1}\|u(s)\|_{L^{q(\Omega)}}^{q} d s+c_{1} \int_{r}^{r+1}\|v(s)\|_{L^{q}(\Gamma)}^{q} d s+2 \int_{r}^{r+1}\|u(s)\|_{1, p}^{p} d s \\
& \leq\left\|u_{0}\right\|_{L^{2}(\bar{\Omega}, d \mu)}^{2} e^{\tau-r}+3 \sup _{r \in \mathbb{R}} \int_{r}^{r+1}\|g(s)\|_{L^{2}(\Omega)}^{2} d s+C .
\end{aligned}
$$

Let $F(s)=\int_{0}^{s} f(\theta) d \theta$, we deduce from (5) that there exist three positive constants $\alpha_{1}, \alpha_{2}$, $\beta$ such that

$$
\alpha_{1}|u|^{q}-\beta \leq F(u) \leq \alpha_{2}|u|^{q}+\beta,
$$

and

$$
\begin{aligned}
& \alpha_{1}|u|_{L^{q(\Omega)}}^{q}-\beta|\Omega| \leq \int_{\Omega} F(u) d x \leq \alpha_{2}|u|_{L^{q(\Omega)}}^{q}+\beta|\Omega|, \\
& \alpha_{1}|v|_{L^{q(\Gamma)}}^{q}-\beta|\Gamma| \leq \int_{\Gamma} F(v) d S \leq \alpha_{2}|v|_{L^{q(\Gamma)}}^{q}+\beta|\Gamma| .
\end{aligned}
$$

Thanks to (34), we deduce from (35)-(36) that

$$
\begin{aligned}
& 2 \int_{r}^{r+1}\|u(s)\|_{W^{1, p}(\Omega)}^{p} d s+\frac{c_{1}}{\alpha_{2}} \int_{r}^{r+1} \int_{\Omega} F(u(s)) d x d s \\
& \quad+\frac{c_{1}}{\alpha_{2}} \int_{r}^{r+1} \int_{\Gamma} F(v(s)) d S d s \\
& \leq\left\|u_{0}\right\|_{L^{2}(\bar{\Omega}, d \mu)}^{2} e^{\tau-r}+3 \sup _{r \in \mathbb{R}} \int_{r}^{r+1}\|g(s)\|_{L^{2}(\Omega)}^{2} d s+C .
\end{aligned}
$$

On the other hand, taking the inner product of (1) with $u_{t}$, we obtain

$$
\begin{aligned}
& \left\|u_{t}\right\|_{L^{2}(\Omega)}^{2}+\left\|v_{t}\right\|_{L^{2}(\Gamma)}^{2}+\frac{d}{d t}\left(\frac{1}{p}\|u\|_{W^{1, p}(\Omega)}^{p}+\int_{\Omega} F(u) d x+\int_{\Gamma} F(v) d S\right) \\
& \leq \frac{1}{2}\|g(s)\|_{L^{2}(\Omega)}^{2}+\frac{1}{2}\left\|u_{t}\right\|_{L^{2}(\Omega)}^{2},
\end{aligned}
$$

which implies

$$
\begin{aligned}
& \left\|u_{t}\right\|_{L^{2}(\Omega)}^{2}+\left\|v_{t}\right\|_{L^{2}(\Gamma)}^{2}+\frac{d}{d t}\left(\frac{2}{p}\|u\|_{W^{1, p}(\Omega)}^{p}+2 \int_{\Omega} F(u) d x+2 \int_{\Gamma} F(v) d S\right) \\
& \quad \leq\|g\|_{L^{2}(\Omega)}^{2} .
\end{aligned}
$$

Combining (37) with (38), by virtue of the uniform Gronwall Lemma 4.6, we get

$$
\begin{aligned}
& \|u(t)\|_{W^{1, p}(\Omega)}^{p}+\int_{\Omega} F(u(t)) d x+\int_{\Gamma} F(v(t)) d S \\
& \quad \leq C\left(\left\|u_{0}\right\|_{L^{2}(\bar{\Omega}, d \mu)}^{2} e^{\tau-r}+\sup _{r \in \mathbb{R}} \int_{r}^{r+1}\|g(s)\|_{L^{2}(\Omega)}^{2} d s+1\right),
\end{aligned}
$$


which implies that for any $\left(u_{0}, \gamma u_{0}\right) \in B$ and $\tau \in \mathbb{R}$, there exists a positive constant $\rho_{2}$ such that

$$
\|u(t)\|_{W^{1, p}(\Omega)}^{p}+\|u(t)\|_{L^{q}(\Omega)}^{q}+\|v(t)\|_{L^{q(\Gamma)}}^{q} \leq C \rho_{2},
$$

where

$$
\begin{aligned}
& \rho_{2}=\sup _{t \in \mathbb{R}} \int_{t}^{t+1}\|g(s)\|_{L^{2}(\Omega)}^{2} d s+1, \\
& \tau_{2}=\max \left\{\tau_{1}, \ln \left(\frac{\left\|u_{0}\right\|_{L^{2}(\bar{\Omega}, d \mu)}^{2}}{\rho_{2}}\right)+\tau\right\} .
\end{aligned}
$$

From Theorem 4.2, the compactness of the Sobolev embedding $W^{1, p}(\Omega) \subset L^{2}(\Omega)$, the compactness of the Sobolev trace embedding $W^{1, p}(\Omega) \subset L^{2}(\Gamma)$ and Lemma 4.2, we have the following result.

Corollary 4.1 The family of processes $\left\{U_{\sigma}(t, \tau)\right\}_{\sigma \in \mathcal{H}_{w}(g)}$ generated by (1)-(5) with initial data $u_{0} \in L^{2}(\bar{\Omega}, d \mu)$ has an $\left(L^{2}(\bar{\Omega}, d \mu), L^{2}(\bar{\Omega}, d \mu)\right)$-uniform (w.r.t. $\left.\sigma \in \mathcal{H}_{w}(g)\right)$ attractor $\mathcal{A}_{2}$, which is compact in $L^{2}(\bar{\Omega}, d \mu)$ and attracts every bounded subset of $L^{2}(\bar{\Omega}, d \mu)$ in the topology of $L^{2}(\bar{\Omega}, d \mu)$. Moreover,

$$
\mathcal{A}_{2}=\omega_{\tau, \mathcal{H}_{w}(g)}\left(B_{0}\right)=\bigcup_{\sigma \in \mathcal{H}_{w}(g)} \mathcal{K}_{\sigma}(s), \quad \forall s \in \mathbb{R},
$$

where $B_{0}$ is the $\left(L^{2}(\bar{\Omega}, d \mu), L^{2}(\bar{\Omega}, d \mu)\right.$ )-uniformly (w.r.t. $\sigma \in \mathcal{H}_{w}(g)$ ) absorbing set in $L^{2}(\bar{\Omega}, d \mu)$ and $\mathcal{K}_{\sigma}(s)$ is the section at $t=s$ of kernel $\mathcal{K}_{\sigma}$ of the process $\left\{U_{\sigma}(t, \tau)\right\}_{\sigma \in \mathcal{H}_{w}(g)}$ with symbol $\sigma \in \mathcal{H}_{w}(g)$.

\subsection{The existence of $\left(L^{2}(\bar{\Omega}, d \mu), L^{q}(\bar{\Omega}, d \mu)\right)$-uniform attractor}

The main purpose of this subsection is to give an asymptotic a priori estimate for the unbounded part of the modular $(|u|,|v|)$ for the solution $(u, v)$ of problem (1)-(5) in the $L^{q}(\bar{\Omega}, d \mu)$-norm.

Theorem 4.3 The family of processes $\left\{U_{\sigma}(t, \tau)\right\}_{\sigma \in \mathcal{H}_{w}(g)}$ corresponding to problem (1)-(5) with initial data $u_{0} \in L^{2}(\bar{\Omega}, d \mu)$ has an $\left(L^{2}(\bar{\Omega}, d \mu), L^{q}(\bar{\Omega}, d \mu)\right.$ )-uniform (w.r.t. $\sigma \in \mathcal{H}_{w}(g)$ ) attractor $\mathcal{A}_{q}$, which is compact in $L^{q}(\bar{\Omega}, d \mu)$ and attracts every bounded subset $B$ of $L^{2}(\bar{\Omega}, d \mu)$ in the topology of $L^{q}(\bar{\Omega}, d \mu)$. Moreover,

$$
\mathcal{A}_{q}=\omega_{\tau, \mathcal{H}_{w}(g)}\left(B_{0}\right)=\bigcup_{\sigma \in \mathcal{H}_{w}(g)} \mathcal{K}_{\sigma}(s), \quad \forall s \in \mathbb{R},
$$

where $B_{0}$ is the $\left(L^{2}(\bar{\Omega}, d \mu), L^{q}(\bar{\Omega}, d \mu)\right)$-uniformly (w.r.t. $\left.\sigma \in \mathcal{H}_{w}(g)\right)$ absorbing set and $\mathcal{K}_{\sigma}(s)$ is the section at $t=s$ of kernel $\mathcal{K}_{\sigma}$ of the process $\left\{U_{\sigma}(t, \tau)\right\}$ with symbol $\sigma \in \mathcal{H}_{w}(g)$.

Proof We need only prove that the process $\left\{U_{\sigma}(t, \tau)\right\}_{\sigma \in \mathcal{H}_{w}(g)}$ satisfies the assumption (ii) of Lemma 4.4. 
From $\left(\mathrm{H}_{3}\right)$, we deduce that for any $\sigma \in \mathcal{H}_{w}(g)$,

$$
\sup _{t \in \mathbb{R}}\|\sigma(t)\|_{L^{2}(\Omega)} \leq \sup _{t \in \mathbb{R}}\|g(t)\|_{L^{2}(\Omega)} \leq K .
$$

Moreover, from Lemma 4.3 and Theorem 4.2, we know that there exists $T_{1}=T_{1}(B, \epsilon, \tau)$ and $M_{0}=M_{0}(\epsilon)$ such that for any $u_{\tau} \in B, t \geq T_{1}$ and $\sigma \in \mathcal{H}_{w}(g)$,

$$
m\left(\Omega\left(\left|U_{\sigma}(t, \tau) u_{\tau}\right| \geq M_{0}\right)\right) \leq \epsilon .
$$

Multiplying (1) with $\left|(u-M)_{+}\right|^{q-1}$ and integrating over $\Omega$, we obtain

$$
\begin{aligned}
& \frac{1}{q} \frac{d}{d t}\left(\left\|(u-M)_{+}\right\|_{L^{q}(\Omega)}^{q}+\left\|(v-M)_{+}\right\|_{L^{q(\Gamma)}}^{q}\right)+(q-1) \int_{\Omega}(u-M)_{+}^{q-2}\left|\nabla(u-M)_{+}\right|^{p} d x \\
& \quad+\int_{\Omega}(u-M)_{+}^{q-1}|u|^{p-2} u d x+\int_{\Omega} f(u)(u-M)_{+}^{q-1} d x \\
& \quad+\int_{\Gamma} f(v)(v-M)_{+}^{q-1} d S \\
& =\int_{\Omega} g(x, t)(u-M)_{+}^{q-1} d x,
\end{aligned}
$$

where $(u-M)_{+}$denotes the positive part of $u-M$, that is,

$$
(u-M)_{+}=\left\{\begin{array}{l}
u-M, \quad u \geq M \\
0, \quad u \leq M
\end{array}\right.
$$

Set $\Omega_{1}=\Omega(|u(t)| \geq M)$ and $\Gamma_{1}=\Gamma(|v(t)| \geq M)$, we have

$$
\begin{aligned}
\frac{1}{q} \frac{d}{d t} & \left(\left\|(u-M)_{+}\right\|_{L^{q}(\Omega)}^{q}+\left\|(v-M)_{+}\right\|_{L^{q}(\Gamma)}^{q}\right)+(q-1) \int_{\Omega_{1}}(u-M)_{+}^{q-2}\left|\nabla(u-M)_{+}\right|^{p} d x \\
& +\int_{\Omega_{1}}(u-M)_{+}^{q-1}|u|^{p-2} u d x+\int_{\Omega_{1}} f(u)(u-M)_{+}^{q-1} d x \\
& +\int_{\Gamma_{1}} f(v)(v-M)_{+}^{q-1} d S \\
= & \int_{\Omega_{1}} g(x, t)(u-M)_{+}^{q-1} d x .
\end{aligned}
$$

Due to (5), we can choose $M \geq M_{0}$ large enough such that

$$
f(u) \geq c u^{q-1} \quad \text { in } \Omega_{1}
$$

for some positive constant $c$. Therefore,

$$
\begin{aligned}
& \frac{1}{q} \frac{d}{d t}\left(\left\|(u-M)_{+}\right\|_{L^{q}(\Omega)}^{q}+\left\|(v-M)_{+}\right\|_{L^{q}(\Gamma)}^{q}\right) \\
& \quad+(q-1) \int_{\Omega_{1}}(u-M)_{+}^{q-2}\left|\nabla(u-M)_{+}\right|^{p} d x
\end{aligned}
$$




$$
\begin{aligned}
& \quad+\int_{\Omega_{1}}(u-M)_{+}^{q-1}|u|^{p-2} u d x+c \int_{\Omega_{1}} u^{q-1}(u-M)_{+}^{q-1} d x \\
& \quad+c \int_{\Gamma_{1}} v^{q-1}(v-M)_{+}^{q-1} d S \\
& \leq \int_{\Omega_{1}} g(x, t)(u-M)_{+}^{q-1} d x .
\end{aligned}
$$

Since

$$
\begin{aligned}
& c \int_{\Omega_{1}} u^{q-1}(u-M)_{+}^{q-1} d x \\
& \quad \geq \frac{c}{2} \int_{\Omega_{1}} u^{q-1}(u-M)_{+}^{q-1} d x+\frac{c}{2} \int_{\Omega_{1}}(u-M)_{+}^{2(q-1)} d x, \\
& c \int_{\Gamma_{1}} v^{q-1}(v-M)_{+}^{q-1} d S \\
& \quad \geq \frac{c}{2} \int_{\Gamma_{1}} v^{q-1}(v-M)_{+}^{q-1} d S+\frac{c}{2} \int_{\Gamma_{1}}(v-M)_{+}^{2(q-1)} d S
\end{aligned}
$$

and

$$
\begin{aligned}
& \int_{\Omega_{1}} g(x, t)(u-M)_{+}^{q-1} d x \\
& \quad \leq \frac{c}{2} \int_{\Omega_{1}}(u-M)_{+}^{2(q-1)} d x+\frac{1}{2 c} \int_{\Omega_{1}}|g(x, t)|^{2} d x .
\end{aligned}
$$

From (42)-(45), we deduce that

$$
\begin{aligned}
\frac{1}{q} \frac{d}{d t}( & \left.\left\|(u-M)_{+}\right\|_{L^{q}(\Omega)}^{q}+\left\|(v-M)_{+}\right\|_{L^{q}(\Gamma)}^{q}\right) \\
& +\frac{c}{2} \int_{\Omega_{1}} u^{q-1}(u-M)_{+}^{q-1} d x+\frac{c}{2} \int_{\Gamma_{1}} v^{q-1}(v-M)_{+}^{q-1} d S \\
\leq & \frac{1}{2 c} \int_{\Omega_{1}}|g(x, t)|^{2} d x .
\end{aligned}
$$

Since $u \geq M$ for all $x \in \Omega_{1}$, we obtain

$$
\begin{aligned}
& \frac{d}{d t}\left(\left\|(u-M)_{+}\right\|_{L^{q(\Omega)}}^{q}+\left\|(v-M)_{+}\right\|_{L^{q(\Gamma)}}^{q}\right) \\
& \quad+c M^{q-2}\left(\left\|(u-M)_{+}\right\|_{L^{q(\Omega)}}^{q}+\left\|(v-M)_{+}\right\|_{L^{q(\Gamma)}}^{q}\right) \\
& \leq C \int_{\Omega_{1}}|g(x, t)|^{2} d x \\
& \leq C \epsilon .
\end{aligned}
$$

It follows from $\left\|\left(u\left(t_{0}\right)-M\right)_{+}\right\|_{L^{q}(\bar{\Omega}, d \mu)}^{q} \leq\left\|u\left(t_{0}\right)\right\|_{L^{q}(\bar{\Omega}, d \mu)}^{q} \leq C$ for any $\tau \in \mathbb{R}, t_{0} \geq \tau_{2}$ and the classical Gronwall inequality that

$$
\left\|(u(t)-M)_{+}\right\|_{L^{q}(\Omega)}^{q}+\left\|(v(t)-M)_{+}\right\|_{L^{q}(\Gamma)}^{q} \leq C \epsilon+\left\|\left(u\left(t_{0}\right)-M\right)_{+}\right\|_{L^{q}(\bar{\Omega}, d \mu)}^{q} e^{c M^{q-2}\left(t_{0}-t\right)},
$$


which implies that for any $\epsilon>0$, there exist two positive constants $M_{1}>M_{0}$ and $\tau_{3} \geq t_{0}$ such that for all $t \geq \tau_{3}$ and $M \geq M_{1}$,

$$
\left\|(u-M)_{+}\right\|_{L^{q}(\Omega)}^{q}+\left\|(v-M)_{+}\right\|_{L^{q}(\Gamma)}^{q} \leq \epsilon .
$$

Repeating the same steps as above, just taking $(u+M)_{-}$instead of $(u+M)_{+}$, we deduce that there exist two positive constants $M_{2}>0$ and $\tau_{4} \geq \tau_{3}$ such that for all $t \geq \tau_{4}$ and $M>M_{2}$,

$$
\left\|(u+M)_{-}\right\|_{L^{q}(\Omega)}^{q}+\left\|(v+M)_{-}\right\|_{L^{q}(\Gamma)}^{q} \leq \epsilon,
$$

where

$$
(u+M)_{-}=\left\{\begin{array}{l}
u+M, \quad u \leq-M \\
0, \quad u \geq-M
\end{array}\right.
$$

Setting $M_{4}=\max \left\{M_{1}, M_{2}\right\}$, we have

$$
\int_{\Omega(|u(t)| \geq M)}(|u|-M)^{q}+\int_{\Gamma(|v(t)| \geq M)}(|v|-M)^{q} \leq \epsilon
$$

for all $t \geq \tau_{4}$ and $M \geq M_{4}$.

Therefore,

$$
\begin{aligned}
& \int_{\Omega(|u(t)| \geq 2 M)}|u|^{q}+\int_{\Gamma(|v(t)| \geq 2 M)}|v|^{q} \\
& =\int_{\Omega(|u(t)| \geq 2 M)}(|u|-M+M)^{q}+\int_{\Gamma(|v(t)| \geq 2 M)}(|v|-M+M)^{q} \\
& \leq 2^{q-1} \int_{\Omega(|u(t)| \geq 2 M)}(|u|-M)^{q}+2^{q-1} \int_{\Gamma(|v(t)| \geq 2 M)}(|v|-M)^{q} \\
& \quad+2^{q-1} \int_{\Omega(|u(t)| \geq 2 M)} M^{q}+2^{q-1} \int_{\Gamma(|v(t)| \geq 2 M)} M^{q} \\
& \leq 2^{q} \int_{\Omega(|u(t)| \geq 2 M)}(|u|-M)^{q}+\int_{\Gamma(|v(t)| \geq 2 M)}(|v|-M)^{q} \\
& \leq 2^{q} \epsilon .
\end{aligned}
$$

\section{$4.4\left(L^{2}(\bar{\Omega}, d \mu),\left(W^{1, p}(\Omega) \cap L^{q}(\Omega)\right) \times L^{q}(\Gamma)\right)$-uniform attractor}

In this subsection, we prove the existence of an $\left(L^{2}(\bar{\Omega}, d \mu),\left(W^{1, p}(\Omega) \cap L^{q}(\Omega)\right) \times L^{q}(\Gamma)\right)$ uniform attractor. For this purpose, we first give some a priori estimates about $\left(u_{t}, v_{t}\right)$ endowed with $L^{2}(\bar{\Omega}, d \mu)$-norm.

Theorem 4.4 Under assumptions $\left(\mathrm{H}_{1}\right)-\left(\mathrm{H}_{3}\right)$, for any bounded subset $B \subset L^{2}(\bar{\Omega}, d \mu)$, any $\tau \in \mathbb{R}$ and $\sigma \in \mathcal{H}_{w}(g)$, there exists a positive constant $T=T(B, \tau) \geq \tau$ such that

$$
\left\|u_{t}(s)\right\|_{L^{2}(\Omega)}^{2}+\left\|v_{t}(s)\right\|_{L^{2}(\Gamma)}^{2} \leq \rho_{3}
$$

for any $u_{\tau} \in B, s \geq T, \sigma \in \mathcal{H}_{w}(g)$, where $\left(u_{t}(s), v_{t}(s)\right)=\left.\frac{d}{d t}\left(U_{\sigma}(t, \tau)\left(u_{0}, \gamma u_{0}\right)\right)\right|_{t=s}$ and $\rho_{3}$ is a positive constant which is independent of $B$ and $\sigma$. 
Proof First, we differentiate (1) and (2) in time, and denoting $\zeta=u_{t}, \eta=v_{t}$, we get

$$
\begin{aligned}
\zeta_{t} & -\operatorname{div}\left(|\nabla u|^{p-2} \nabla \zeta\right)-(p-2) \operatorname{div}\left(|\nabla u|^{p-4}(\nabla u \cdot \nabla \zeta) \nabla u\right) \\
& +(p-1)|u|^{p-2} \zeta+f^{\prime}(u) \zeta=\frac{d g}{d t}, \\
\eta_{t} & +(p-2)|\nabla v|^{p-4}(\nabla v \cdot \nabla \eta) \frac{\partial v}{\partial v}+|\nabla v|^{p-2} \frac{\partial \eta}{\partial v}+f^{\prime}(v) \eta=0,
\end{aligned}
$$

where '.' denotes the dot product in $\mathbb{R}^{n}$.

Multiplying (46) by $\zeta$ and integrating over $\Omega$, and combining (4) with (47), we obtain

$$
\begin{aligned}
& \frac{1}{2} \frac{d}{d t}\left(\|\zeta\|_{L^{2}(\Omega)}^{2}+\|\eta\|_{L^{2}(\Gamma)}^{2}\right)+\int_{\Omega}|\nabla u|^{p-2}|\nabla \zeta|^{2} d x \\
& \quad+(p-2) \int_{\Omega}|\nabla u|^{p-4}(\nabla u \cdot \nabla \zeta)^{2} d x+(p-1) \int_{\Omega}|u|^{p-2}|\zeta|^{2} d x \\
& \quad \leq l\left(\|\zeta\|_{L^{2}(\Omega)}^{2}+\|\eta\|_{L^{2}(\Gamma)}^{2}\right)+\left\|\frac{d g}{d t}\right\|_{L^{2}(\Omega)}\|\zeta\|_{L^{2}(\Omega)} .
\end{aligned}
$$

On the other hand, for any $r \geq \tau$, integrating (38) from $r$ to $r+1$ and using (39), we find

$$
\begin{aligned}
& \int_{r}^{r+1}\|\zeta(s)\|_{L^{2}(\Omega)}^{2}+\int_{r}^{r+1}\|\eta(s)\|_{L^{2}(\Gamma)}^{2} \\
& \quad \leq C\left(\left\|u_{0}\right\|_{L^{2}(\bar{\Omega}, d \mu)}^{2} e^{\tau-r}+\sup _{r \in \mathbb{R}} \int_{r}^{r+1}\|g(s)\|_{L^{2}(\Omega)}^{2} d s+1\right) .
\end{aligned}
$$

Therefore, we deduce from the uniformly Gronwall inequality that

$$
\begin{aligned}
& \left\|u_{t}(s)\right\|_{L^{2}(\Omega)}^{2}+\left\|v_{t}(s)\right\|_{L^{2}(\Gamma)}^{2} \\
& \quad \leq C\left(\left\|u_{0}\right\|_{L^{2}(\bar{\Omega}, d \mu)}^{2} e^{\tau-r}+\sup _{r \in \mathbb{R}} \int_{r}^{r+1}\|g(s)\|_{L^{2}(\Omega)}^{2} d s+1+\int_{t-1}^{t}\left\|\frac{d g}{d t}\right\|_{L^{2}(\Omega)}^{2}\right),
\end{aligned}
$$

which implies that there exist two positive constants $\tau_{5} \geq \tau$ and a positive constant $\rho_{3}$ such that

$$
\left\|u_{t}(s)\right\|_{L^{2}(\Omega)}^{2}+\left\|v_{t}(s)\right\|_{L^{2}(\Gamma)}^{2} \leq \rho_{3}
$$

for any $\left(u_{0}, \gamma u_{0}\right) \in B, \tau \in \mathbb{R}$ and $t \geq \tau_{5}$, where

$$
\rho_{3}=C\left(\sup _{r \in \mathbb{R}} \int_{r}^{r+1}\|g(s)\|_{L^{2}(\Omega)}^{2} d s+1+\int_{t-1}^{t}\left\|\frac{d g}{d t}\right\|_{L^{2}(\Omega)}^{2}\right) .
$$

Next, we prove the process $\left\{U_{\sigma}(t, \tau)\right\}_{\sigma \in \mathcal{H}_{w}(g)}$ is uniformly (w.r.t. $\sigma \in \mathcal{H}_{w}(g)$ ) asymptotically compact in $\left(W^{1, p}(\Omega) \cap L^{q}(\Omega)\right) \times L^{q}(\Gamma)$.

Theorem 4.5 Assume that $f$ and $g$ satisfy $\left(\mathrm{H}_{1}\right)-\left(\mathrm{H}_{3}\right)$. Then the family of processes $\left\{U_{\sigma}(t, \tau)\right\}_{\sigma \in \mathcal{H}_{w}(g)}$ corresponding to problem (1)-(5) with initial data $u_{0} \in L^{2}(\bar{\Omega}, d \mu)$ is $\left(L^{2}(\bar{\Omega}, d \mu),\left(W^{1, p}(\Omega) \cap L^{q}(\Omega)\right) \times L^{q}(\Gamma)\right)$-uniformly (w.r.t. $\left.\sigma \in \mathcal{H}_{w}(g)\right)$ asymptotically compact, i.e., there exists a compact uniformly attracting set in $\left(W^{1, p}(\Omega) \cap L^{q}(\Omega)\right) \times L^{q}(\Gamma)$, which attracts any bounded subset $B \subset L^{2}(\bar{\Omega}, d \mu)$ in the topology of $\left(W^{1, p}(\Omega) \cap L^{q}(\Omega)\right) \times L^{q}(\Gamma)$. 
Proof Let $B_{0}$ be an $\left(L^{2}(\bar{\Omega}, d \mu),\left(W^{1, p}(\Omega) \cap L^{q}(\Omega)\right) \times L^{q}(\Gamma)\right)$-uniformly (w.r.t. $\sigma \in \mathcal{H}_{w}(g)$ ) absorbing set obtained in Theorem 4.2, then we need only to show that for any $\left\{u_{\tau_{n}}\right\} \subset B_{0}$, $\left\{\sigma_{n}\right\} \subset \mathcal{H}_{w}(g)$ and $t_{n} \rightarrow \infty,\left\{U_{\sigma_{n}}\left(t_{n}, \tau_{n}\right) u_{\tau_{n}}\right\}_{n=1}^{\infty}$ is pre-compact in $\left(W^{1, p}(\Omega) \cap L^{q}(\Omega)\right) \times L^{q}(\Gamma)$.

Thanks to Lemma 4.2, it is sufficient to verify that for any $\left\{u_{\tau_{n}}\right\} \subset B_{0},\left\{\sigma_{n}\right\} \subset \mathcal{H}_{w}(g)$ and $t_{n} \rightarrow \infty,\left\{U_{\sigma_{n}}\left(t_{n}, \tau_{n}\right) u_{\tau_{n}}\right\}_{n=1}^{\infty}$ is pre-compact in $W^{1, p}(\Omega)$.

In fact, from Corollary 4.1 and Theorem 4.3 , we know that $\left\{U_{\sigma_{n}}\left(t_{n}, \tau_{n}\right) u_{\tau_{n}}\right\}_{n=1}^{\infty}$ is precompact in $L^{2}(\bar{\Omega}, d \mu)$ and $L^{q}(\bar{\Omega}, d \mu)$.

Without loss of generality, we assume that $\left\{U_{\sigma_{n}}\left(t_{n}, \tau_{n}\right) u_{\tau_{n}}\right\}_{n=1}^{\infty}$ is a Cauchy sequence in $L^{2}(\bar{\Omega}, d \mu)$ and $L^{q}(\bar{\Omega}, d \mu)$.

Now, we prove that $\left\{U_{\sigma_{n}}\left(t_{n}, \tau_{n}\right) u_{\tau_{n}}\right\}_{n=1}^{\infty}$ is a Cauchy sequence in $W^{1, p}(\Omega)$.

Denote by $u_{n}^{\sigma_{n}}\left(t_{n}\right):=U_{\sigma_{n}}\left(t_{n}, \tau_{n}\right) u_{\tau_{n}}$, we deduce from Lemma 2.2 that

$$
\begin{aligned}
\left\|u_{n}^{\sigma_{n}}\left(t_{n}\right)-u_{m}^{\sigma_{m}}\left(t_{m}\right)\right\|_{W^{1, p}(\Omega)}^{p} & \\
\leq & \left(-\frac{d}{d t} u_{n}^{\sigma_{n}}\left(t_{n}\right)-f\left(u_{n}^{\sigma_{n}}\left(t_{n}\right)\right)+\frac{d}{d t} u_{m}^{\sigma_{m}}\left(t_{m}\right)+f\left(u_{m}^{\sigma_{m}}\left(t_{m}\right)\right), u_{n}^{\sigma_{n}}\left(t_{n}\right)-u_{m}^{\sigma_{m}}\left(t_{m}\right)\right) \\
& \quad+\left(-\frac{d}{d t} v_{n}^{\sigma_{n}}\left(t_{n}\right)-f\left(v_{n}^{\sigma_{n}}\left(t_{n}\right)\right)+\frac{d}{d t} v_{m}^{\sigma_{m}}\left(t_{m}\right)+f\left(v_{m}^{\sigma_{m}}\left(t_{m}\right)\right), v_{n}^{\sigma_{n}}\left(t_{n}\right)-v_{m}^{\sigma_{m}}\left(t_{m}\right)\right) \\
= & I_{1}+I_{2} .
\end{aligned}
$$

We now estimate separately the two terms $I_{1}$ and $I_{2}$. By simple calculations and the Hölder inequality, we deduce that

$$
\begin{aligned}
I_{1} \leq & \left\|\frac{d}{d t} u_{n}^{\sigma_{n}}\left(t_{n}\right)-\frac{d}{d t} u_{m}^{\sigma_{m}}\left(t_{m}\right)\right\|_{L^{2}(\Omega)}\left\|u_{n}^{\sigma_{n}}\left(t_{n}\right)-u_{m}^{\sigma_{m}}\left(t_{m}\right)\right\|_{L^{2}(\Omega)} \\
& +C\left(1+\left\|u_{n}^{\sigma_{n}}\left(t_{n}\right)\right\|_{L^{q}(\Omega)}^{q-1}+\left\|u_{m}^{\sigma_{m}}\left(t_{m}\right)\right\|_{L^{q}(\Omega)}^{q-1}\right)\left\|u_{n}^{\sigma_{n}}\left(t_{n}\right)-u_{m}^{\sigma_{m}}\left(t_{m}\right)\right\|_{L^{q}(\Omega)}
\end{aligned}
$$

and

$$
\begin{aligned}
I_{2} \leq & \left\|\frac{d}{d t} v_{n}^{\sigma_{n}}\left(t_{n}\right)-\frac{d}{d t} v_{m}^{\sigma_{m}}\left(t_{m}\right)\right\|_{L^{2}(\Gamma)}\left\|v_{n}^{\sigma_{n}}\left(t_{n}\right)-v_{m}^{\sigma_{m}}\left(t_{m}\right)\right\|_{L^{2}(\Gamma)} \\
& +C\left(1+\left\|v_{n}^{\sigma_{n}}\left(t_{n}\right)\right\|_{L^{q}(\Gamma)}^{q-1}+\left\|v_{m}^{\sigma_{m}}\left(t_{m}\right)\right\|_{L^{q}(\Gamma)}^{q-1}\right)\left\|v_{n}^{\sigma_{n}}\left(t_{n}\right)-v_{m}^{\sigma_{m}}\left(t_{m}\right)\right\|_{L^{q}(\Gamma)},
\end{aligned}
$$

which combining with Corollary 4.1, Theorem 4.3 and Theorem 4.4 yields Theorem 4.5 immediately.

Competing interests

The authors declare that they have no competing interests.

Authors' contributions

All authors typed, read and approved the final manuscript.

\section{Author details}

${ }^{1}$ Department of Basic, Henan Mechanical and Electrical Engineering College, Xinxiang, 453003, P.R. China. ${ }^{2}$ School of Mathematics and Statistics, Xi'an Jiaotong University, Xi'an, 710049, P.R. China.

\section{Acknowledgements}

The authors would like to thank the referees for their valuable suggestions. 


\section{References}

1. Arrieta, JM, Carvalho, AN, Bernal, AR: Attractors of parabolic problems with nonlinear boundary conditions uniform bounds. Partial Differ. Equ. 25, 1-37 (2000)

2. Bernal, AR: Attractors for parabolic equations with nonlinear boundary conditions, critical exponents and singular initial data. J. Differ. Equ. 181, 165-196 (2002)

3. Fan, ZH, Zhong, CK: Attractors for parabolic equations with dynamic boundary conditions. Nonlinear Anal. 68 , 1723-1732 (2008)

4. Yang, L: Uniform attractors for the closed process and applications to the reaction-diffusion with dynamical boundary condition. Nonlinear Anal. 71, 4012-4025 (2009)

5. Chepyzhov, VV, Vishik, Ml: Attractors for Equations of Mathematical Physics. Am. Math. Soc., Providence (2002)

6. Arrieta, JM, Carvalho, AN, Bernal, AR: Parabolic problems with nonlinear boundary conditions and critical nonlinearities. J. Differ. Equ. 156, 376-406 (1999)

7. Constantin, A, Escher, J: Global existence for fully parabolic boundary value problems. Nonlinear Differ. Equ. Appl. 13, 91-118 (2006)

8. Constantin, A, Escher, J, Yin, Z: Global solutions for quasilinear parabolic systems. J. Differ. Equ. 197, $73-84$ (2004)

9. Petersson, J: A note on quenching for parabolic equations with dynamic boundary conditions. Nonlinear Anal. 58, 417-423 (2004)

10. Popescu, L, Bernal, AR: On a singularly perturbed wave equation with dynamical boundary conditions. Proc. R. Soc. Edinb., Sect. A, Math. 134, 389-413 (2004)

11. Yang, L, Yang, MH, Kloeden, PE: Pullback attractors for non-autonomous quasi-linear parabolic equations with a dynamical boundary condition. Discrete Contin. Dyn. Syst., Ser. B 17, 2635-2651 (2012)

12. Anh, CT, Ke, TD: On quasilinear parabolic equations involving weighted $p$-Laplacian operators. Nonlinear Differ. Equ. Appl. 17, 195-212 (2010)

13. Bartsch, T, Liu, Z: On a superlinear elliptic p-Laplacian equation. J. Differ. Equ. 198, 149-175 (2004)

14. Chen, GX: Uniform attractors for the non-autonomous parabolic equation with nonlinear Laplacian principal part in unbounded domain. Differ. Equ. Appl. 2(1), 105-121 (2010)

15. Fernandez, BJ, Rossi, JD: Existence results for the $p$-Laplacian with nonlinear boundary conditions. J. Math. Anal. Appl. 263, 195-223 (2001)

16. Martinez, S, Rossi, JD: Weak solutions for the $p$-Laplacian with a nonlinear boundary condition at resonance. Electron. J. Differ. Equ. 27, 1-14 (2003)

17. Martinez, SR, Rossi, JD: Isolation and simplicity for the first eigenvalue of the $p$-Laplacian with a nonlinear boundary conditions. Abstr. Appl. Anal. 7, 287-293 (2002)

18. Yin, Z: Global existence for elliptic equations with dynamic boundary conditions. Arch. Math. 81, $567-574$ (2003)

19. Martinez, SR, Rossi, JD: On the Fučik spectrum and a resonance problem for the $p$-Laplacian with a nonlinear boundary condition. Nonlinear Anal. 59, 813-848 (2004)

20. Chen, GX, Zhong, CK: Uniform attractors for non-autonomous p-Laplacian equations. Nonlinear Anal. 68, 3349-3363 (2008)

21. You, B, Zhong, CK: Global attractors for $p$-Laplacian equations with dynamic flux boundary conditions. Adv. Nonlinear Stud. 13, 391-410, (2013)

22. Robinson, JC: Infinite-Dimensional Dynamical Systems. Cambridge University Press, Cambridge (2001)

23. Adams, RA, Fournier, JJF: Sobolev Spaces. Academic Press, Amsterdam (2003)

24. Lê, A: Eigenvalue problems for the $p$-Laplacian. Nonlinear Anal. 64, 1057-1099 (2006)

25. Temam, R: Infinite-Dimensional Systems in Mechanics and Physics. Springer, New York (1997)

26. Babin, AV, Vishik, MI: Attractors of Evolution Equations. North-Holland, Amsterdam (1992)

27. Lu, SS, Wu, HQ, Zhong, CK: Attractors for nonautonomous 2D Navier-Stokes equations with normal external forces. Discrete Contin. Dyn. Syst. 13(3), 701-719 (2005)

28. Li, Y, Zhong, CK: Pullback attractor for the norm-to-weak continuous process and application to the non-autonomous reaction-diffusion equations. Appl. Math. Comput. 190, 1020-1029 (2007)

doi:10.1186/1687-2770-2013-128

Cite this article as: Li and You: Uniform attractors for the non-autonomous $p$-Laplacian equations with dynamic flux boundary conditions. Boundary Value Problems 2013 2013:128.

\section{Submit your manuscript to a SpringerOpen ${ }^{\circ}$ journal and benefit from:}

- Convenient online submission

Rigorous peer review

- Immediate publication on acceptance

Open access: articles freely available online

- High visibility within the field

- Retaining the copyright to your article 